\title{
A super-Alfvénic model of dark clouds
}

\author{
Paolo Padoan \\ Instituto Nacional de Astrofísica, Óptica y Electrónica, Apartado Postal 216, 72000 Puebla, México \\ Åke Nordlund \\ Astronomical Observatory and Theoretical Astrophysics Center, Juliane Maries Vej 30, DK-2100 Copenhagen, \\ Denmark
}

\begin{abstract}
Supersonic random motions are observed in dark clouds and are traditionally interpreted as Alfvén waves, but the possibility that these motions are super-Alfvénic has not been ruled out.

In this work we report the results of numerical experiments in two opposite regimes; $\mathcal{M}_{A} \sim 1$ and $\mathcal{M}_{A} \gg 1$, where $\mathcal{M}_{A}$ is the initial Alfvénic Mach number - the ratio of the rms velocity to the Alfvén speed. Our results show that models with $\mathcal{M}_{A} \gg 1$ are consistent with the observed properties of molecular clouds that we have tested statistics of extinction measurements, Zeeman splitting measurements of magnetic field strength, line width versus integrated antenna temperature of molecular emission line spectra, statistical $B-n$ relation, and scatter in that relation- while models with $\mathcal{M}_{A} \sim 1$ have properties that are in conflict with the observations.

We find that both the density and the magnetic field in molecular clouds may be very intermittent. The statistical distributions of magnetic field and gas density are related by a power law, with an index that decreases with time in experiments with decaying turbulence. After about one dynamical time it stabilizes at $B \propto n^{0.4}$. Magnetically dominated cores form early in the evolution, while later on the intermittency in the density field wins out, and also cores with weak field can be generated, by mass accretion along magnetic field lines.
\end{abstract}

Subject headings: turbulence - ISM: kinematics and dynamics- magnetic field 


\section{Introduction}

The observation of supersonic motions in molecular clouds (eg Zuckerman \& Palmer 1974) raised the question of how these motions could be supported (Norman \& Silk 1980; Fleck 1981; Scalo \& Pumphrey 1982). Supersonic motions are expected to quickly dissipate their energy in highly radiative shocks, because of the very short cooling time of molecular gas or metal rich atomic gas (Mestel \& Spitzer 1956; Spitzer 1968; Goldreich \& Kwan 1974).

Strictly related was the issue of the support of molecular clouds (MCs) against gravitational collapse, since it was soon realized that the observed motions could not be understood as a gravitational collapse (Zuckerman \& Evans 1974; Morris et al. 1974), although MCs contain many Jeans' masses.

Theoreticians therefore formulated the hypothesis that MCs were primarily magnetically supported (Mestel 1965, Strittmatter 1966; Parker 1973; Mouschovias 1976a,b; McKee \& Zweibel 1995) and interpreted the observed motions as long-wavelength hydro-magnetic waves (Arons \& Max 1975; Zweibel \& Josafatsson 1983; Elmegreen 1985, Falgarone \& Puget 1986). It was also shown that the properties of the observed 'turbulence' (Larson 1981; Leung, Kutner \& Mead 1982; Myers 1983; Quiroga 1983; Sanders, Scoville \& Solomon 1985; Goldsmith \& Arquilla 1985; Dame et al. 1986; Falgarone \& Pérault 1987) could be understood if the motions were sub-Alfvénic (Myers \& Goodman 1988; Mouschovias \& Psaltis 1995, Xie 1997).

Nevertheless, recent attempts to detect the Zeeman effect, in lines of molecules such as $\mathrm{OH}$ and $\mathrm{CN}$, that probe regions of dense gas (Crutcher et al. 1993; Crutcher et al. 1996), resulted in a number of nondetections of the effect, and therefore in rather stringent upper limits for the magnetic field strength, despite the fact that regions expected to favor detections were targeted. It is therefore possible, that the field strength in dense molecular gas is weaker than assumed in theoretical studies.

In the present work, we study the dynamics of models that share some properties with molecular clouds, using numerical solutions of the equations of 3-D compressible magneto-hydrodynamics (MHD) in a regime of highly supersonic random motions. We idealize the system by omitting self gravity, ambipolar diffusion, radiative energy transfer, etc., and concentrate instead on the aspects that are directly related to the random, supersonic nature of the motions. The main limitation of our numerical models, compared with MCs, is the absence of gravity. We have excluded gravity on purpose (our code is capable of handling self-gravity), because one of the aims of our work is to show that (magneto-)hydrodynamic processes alone are able to explain many of the observed properties of MCs. Although gravity is certainly responsible for the final collapse of high density regions into stars, we suspect that supersonic random motions are responsible for setting up many of the properties that characterize molecular clouds, with only minor contributions from gravity (Padoan 1995; Padoan, Jones \& Nordlund 1997; Padoan, Nordlund \& Jones 1997, Padoan et al., 1998b. The importance of supersonic motions in fragmenting the gas is certainly apparent on small scales, where young and probably transient clumps are not originated by gravitational instability (Falgarone, Puget \& Pérault 1992; Langer et al. 1995).

Previous numerical studies have shown that compressible turbulence can qualitatively explain several observational properties of MCs, even if the effect of gravity and magnetic fields are not included. The first two-dimensional (2-D) simulations of turbulence with rms Mach number larger than one were performed by Passot \& Pouquet (1987). These were the first simulations where shocks were shown to develop inside a turbulent flow. It was immediately recognized that shocks might have been responsible for the fragmentation of the density field inside MCs, and especially for the origin of their filamentary structure (Passot, Pouquet \& Woodward 1988). The importance of shock formation inside mildly supersonic flows was later confirmed in 3-D simulations by Lee, Lele \& Moin (1991). Kimura \& Tosa (1993) simulated the passage of a strong shock through a turbulent molecular cloud, and found that this process can generate dense clumps, with a power law mass spectrum. Vázquez-Semadeni (1994) made use of 2-D numerical simulations to show that supersonic turbulence generates a very intermittent density field, reminiscent of the clumpy nature of MCs. The density field was also found to be self-similar, which could be the reason for the hierarchical structure of MCs (Scalo 1985, Houlahan \& Scalo 1992, Elmegreen \& Falgarone 1996, Elmegreen 1997). Falgarone et al. (1994), analyzing the numerical simulation by Porter, Pouquet \& Woodward (1994), argued that the properties of the profiles of molecular emission spectra from MCs can 
be interpreted as arising from turbulent motions.

Other numerical works included gravity in the turbulent flows, yet without describing the magnetic field. Turbulence was shown to be able to prevent gravitational collapse (cf Chandrasekhar 1958; Arny 1971; Bonazzola et al. 1987, 1992) in the 2-D numerical simulations by Léorat, Passot \& Pouquet (1990). Vázquez-Semadeni, Passot \& Pouquet (1995) modeled the galactic disc, on the scale of $1 \mathrm{Kpc}$, as a turbulent self-gravitating flow. They simulate a two dimensional turbulent flow that is forced by the energy released by star formation (expansions of HII regions), and found that the main mechanism of cloud formation is the turbulent ram pressure, rather than gravity. They were not able to form self gravitating clouds, due to limitations in the thermal modeling, and the consequent low density contrast.

A three dimensional description of a magnetized self-gravitating cloud was given by Carlberg \& $\mathrm{Pu}-$ dritz (1990), and was used to simulate molecular emission spectra by Stenholm \& Pudritz (1993). Carlberg \& Pudritz found that the magnetic field and hydromagnetic waves can support the cloud against gravity. The clouds contract, because of ambipolar diffusion, on a time scale of approximately four freefall times. These simulations do not solve the MHD equations, but instead make use of a 'sticky particles' code. Energy is injected in the form of a spectrum of Alfvén waves, and the outcome of the computation is dependent on the spectral index, that is a free parameter. This way of forcing the particles is rather unphysical, because an arbitrary spectrum of Alfvén waves is imposed, instead of being obtained as a result of the simulated magneto-hydrodynamics. Passot, Vázquez-Semadeni \& Pouquet (1995) introduced the magnetic field in their previous two dimensional model for the galactic disc (Vázquez-Semadeni, Passot \& Pouquet 1995), and obtained a flow with rough equipartition of kinetic and magnetic energy, probably in rough equipartition also with the mean thermal energy. The same simulation, and others with larger density contrast and resolution, have been studied by Vázquez-Semadeni, Ballesteros-Paredes \& Rodríguez (1997), who were able to reproduce the observed relation between line-width and size (Larson 1981). Gammie and Ostriker (1996) solved the MHD equations in a slab geometry, including self-gravity. By forcing the flow with a nonlinear spectrum of MHD waves, they were able to prevent the gravitational collapse of their one dimensional cloud model.
Apart from the intentional exclusion of gravity, we differ from previous studies of turbulent flows in several respects. First of all we have solved the MHD equations in three dimensions, while all previous solutions are in two or one dimensions. In MHD the dimensionality of the flow has a fundamental importance, because it determines the topological freedom of the magnetic field. A price that has to be paid for using fully three-dimensional simulations is a significant reduction of the number of points in each spatial direction. Thus, while our $128^{3}$ runs correspond to $\sim 210^{6}$ degrees of freedom, two dimensional simulation such as those of Vázquez-Semadeni et al. (1997), that use up to 800 points in each direction, still need less than a third of the computing resources of our three dimensional simulations. Studies of varying dimensionality are thus complementary, and may contribute to uncover different aspects of the system under study.

An important property of our models is the high Mach numbers of the flows (rms up to $\sim 30$, maximum $\sim 60$ ), that allows us to investigate the super-sonic regime of interstellar medium flows on rather large scales, up to about 40 pc. Previous works have normally been limited to smaller rms Mach number. For example some values of the initial rms Mach number in decaying flows are: $\mathcal{M}_{i}=4$ in the two dimensional experiment by Passot, Pouquet \& Woodward (1988); $\mathcal{M}_{i}=1$ in three dimensional runs by Porter, Pouquet \& Woodward $1992 ; \mathcal{M}_{i}=10$ in one dimensional simulations by Gammie \& Ostriker (1996); $\mathcal{M}_{i}=5$ in MacLow et al. (1998) in three dimensions. Other values of rms Mach number in driven numerical experiments are: $\mathcal{M}=3$ in three dimensional simulations by Lee, Lele \& Moin (1991); $\mathcal{M}=3$ in numerical experiments by Scalo et al. (1998); $\mathcal{M}=5$ in three dimensional simulations by Stone, Ostriker \& Gammie (1998). The two dimensional simulations by Vázquez-Semadeni, Passot \& Pouquet (1996) have large Mach numbers because of the cooling they include.

While previous models of magnetized clouds focused on the role of the magnetic field as opposed to gravity, and therefore have assumed a magnetic pressure much larger than the gas pressure, the main purpose of the present work is to show that MCs are well described as flows with lower magnetic pressure than previously assumed.

In this work we report the results of numerical experiments in two opposite regimes; $\mathcal{M}_{A} \gg 1$ and 
$\mathcal{M}_{A} \sim 1$, where $\mathcal{M}_{A}$ is the initial Alfvénic Mach number -the ratio of the rms velocity to the Alfvén speed. We argue that the observations of magnetic field strengths are consistent with a scenario where the mean magnetic pressure is dynamically low; i.e., where $\mathcal{M}_{A}^{2} \gg 1$. Super-sonic and super-Alfvénic magneto-hydrodynamic (MHD) flows develop a very intermittent spatial distribution of the magnetic energy, such that when the field is detected at a favorable position, its estimated strength is far higher than the mean field strength. Therefore, dense cores with sub-Alfvénic velocity dispersion can be generated in super-Alfvénic flows, in agreement with the observations.

\section{The experiments}

The st idy of the dynamics of MCs belongs to the field of random and super-sonic MHD flows. The Reynolds number and the magnetic Reynolds number in MC flows are very large. The random nature is therefore a basic feature of the dynamics of these flows, and requires an appropriate description.

A realistic description of the dynamics of molecular clouds have to be based on the numerical solution of the compressible MHD equations in three dimensions, in a regime of random and highly supersonic motions. We solve the compressible MHD equations in such a regime, at a numerical resolution of $128^{3}$, with a code designed for turbulence and MHD turbulence experiments (Nordlund, Galsgaard \& Stein 1994; Stein, Galsgaard \& Nordlund 1994; Galsgaard \& Nordlund 1996; Nordlund, Stein \& Galsgaard 1996; Nordlund \& Galsgaard 1997), specifically adapted to be able to deal with very strong shocks and very large density contrasts.

Although we have already developed a version of the code with the inclusion of the gravitational force, all the experiments were run without gravity, for the following reasons:

- We are interested in studying if gas dynamics and magneto-hydrodynamics alone can shape distributions that are similar to those observed, even without invoking gravity.

- The observed motions have velocities comparable with the virial velocity, or larger, on a range of scales, and the clouds are not free-falling.

- If the results of our experiments are discussed only up to a time shorter than or comparable to the dynamical (or free-fall) time, all our conclusions remain basically unchanged. This time is about a few million years on a scale of $10 \mathrm{pc}$, and clouds are not supposed to live much longer than that, before star formation takes place and becomes energetically important.

We note that the fact that clouds are observed to have velocities comparable to virial velocities, and are observed to not be free-falling, are not acceptable justifications for neglecting self-gravity; a complete model of interstellar clouds should instead explain why they are not free-falling even in the presence of gravity. However, by concentrating first, in the present paper, on a situation where gravity is neglected, we provide a point of reference for subsequent studies, where we do include self-gravity (Padoan et al., 1999b).

\subsection{The equations}

We solve the compressible MHD equations:

$$
\begin{gathered}
\frac{\partial \ln \rho}{\partial t}+\mathbf{v} \cdot \nabla \ln \rho=-\nabla \cdot \mathbf{v} \\
\frac{\partial \mathbf{v}}{\partial t}+\mathbf{v} \cdot \nabla \mathbf{v}=-\frac{P}{\rho} \nabla \ln P+\frac{1}{\rho} \mathbf{j} \times \mathbf{B}+\mathbf{f} \\
\frac{\partial e}{\partial t}+\mathbf{v} \cdot \nabla e=-\frac{P}{\rho} \nabla \cdot \mathbf{v}+Q_{\text {dissipation }}+Q_{\text {radiation }} \\
\frac{\partial \mathbf{B}}{\partial t}=\nabla \times \mathbf{v} \times \mathbf{B} \\
\mathbf{j}=\nabla \times \mathbf{B}
\end{gathered}
$$

plus numerical diffusion terms, and with periodic boundary conditions. $\mathbf{v}$ is the velocity, $\mathbf{B}$ the magnetic field, $\mathbf{f}$ an external force $(=0$ in these particular experiments), and $P=\rho T$ is the pressure at $T \approx$ const.

Conditions in the cold molecular clouds that we are modeling are such that an isothermal approximation is adequate; the radiative heat exchange is so efficient that the temperature remains low in most places. Even if the temperature momentarily increases in shocks, the subsequent cooling is rapid, and the result is shock structures that are qualitatively and quantitatively similar to isothermal shocks. 
We have thus used isothermal conditions in most of our runs and have verified that this is appropriate, by rerunning segments of some experiments using the full energy equation. No significant change of the statistics was found and, since using the full energy equation increases the cost of the experiments considerably (the strong cooling required to maintain a low temperature forces a much smaller time step), we performed most of the experiments at constant temperature.

The absence of an explicit resistivity $\eta$ in the induction equation corresponds to an assumption of flux freezing on well resolved scales. The code uses shock and current sheet capturing techniques to ensure that magnetic and viscous dissipation at the smallest resolved scales provide the necessary dissipation paths for magnetic and kinetic energy. As shown by Galsgaard \& Nordlund (1996, 1997), dissipation of magnetic energy in highly turbulent, compressible MHD plasmas occurs at a rate that is independent of the details of the small scale dissipation. In ordinary hydrodynamic turbulence the corresponding property is one of the cornerstones of Kolmogorov (1941) scaling.

The works by Galsgaard \& Nordlund (1996, 1997) refer to compressible flows, but since the ratio of mean kinetic energy to mean magnetic energy is small, motions are in practice roughly incompressible, at least in the directions perpendicular to the local magnetic field. In the case referred to in the present paper as Model A, the mean kinetic energy is instead large compared to the mean magnetic energy, and the situation might be rather different, and more similar to Burger's turbulence than both classical Kolmogorov turbulence, and the situation studied by Galsgaard and Nordlund. However, Burger's turbulence is also characterized by having a dissipation that is, in the limit, independent of the value of the viscosity (cf. Saffman 1968, p. 485, sec. 6).

We have not included ambipolar diffusion in any of the driven experiments. The value of the rms Mach number in the driven experiments is comparable to the value estimated in molecular clouds on a scale range from $3 \mathrm{pc}$ to $50 \mathrm{pc}$. On such scales, ambipolar diffusion occurs in a time significantly longer than the dynamical time, as recently shown by Myers \& Khersonsky (1995) and is expected to be of secondary importance (see also Elmegreen \& Fiebig 1993).

On sufficiently small scales and high densities, ambipolar diffusion can have significant effects (Zweibel \& Brandenburg 1994, 1997; Tagger et al1995). Since the decay runs have lower rms Mach number (smaller scale), and since ambipolar diffusion is considered an important dissipation mechanism in magnetized clouds (McKee et al. 1993), the ambipolar drift has been included in some of the decaying runs.

\subsection{The code}

The code solves the compressible MHD equations on a 3D staggered mesh, with volume centered mass density and thermal energy, face centered velocity and magnetic field components, and edge centered electric currents and electric fields (Nordlund, Stein \& Galsgaard 1996).

The original code works with "per-unit-volume" variables; mass density, momenta, and thermal energy per unit volume. In the super-sonic regime relevant in the present application, we found it advantageous to rewrite the code in terms of "per-unit-mass" variables; $\ln \rho, u$, and $E=\frac{3}{2} \frac{P}{\rho}$. With these variables, the time evolution of all variables is governed by equations of the type

$$
\frac{D f}{D t}=\frac{\partial f}{\partial t}+\mathbf{v} \cdot \nabla f=\ldots
$$

i.e., equations that specify the time rate of change following the motion. These are better conditioned than the divergence type equations that result from using per-unit-volume variables (the large - order $M^{2}$ density variation in isothermal shocks cause the perunit-volume fluxes to vary over several orders of magnitude).

We use spatial derivatives accurate to 6 th order, interpolation accurate to 5th order, and Hyman's 3rd order time stepping method (Hyman 1979).

In order to minimize the viscous and resistive influence on well resolved scales, we use monotonic 3rd order hyper-diffusive fluxes instead of normal diffusive fluxes, and in order to capture hydrodynamic and magneto-hydrodynamic shocks we add diffusivities proportional to the negative part of the velocity divergence, and resistivity proportional to the negative part of the cross-field (two-dimensional) velocity divergence. Further details of the numerical methods are given by Nordlund, Stein \& Galsgaard (1996) and Nordlund \& Galsgaard (1997).

\subsection{Weak and strong magnetic field}

For the purpose of this work we have run several experiments, that can be divided into two main groups: 
i) super-Alfvénic runs (models A), with $\mathcal{M}_{A, i} \approx 10$ (in one case $\mathcal{M}_{A, i} \approx 30$ ), and $\beta_{i} \geq 1$; ii) equipartition (of kinetic and magnetic energies) runs (models B), with $\mathcal{M}_{A, i} \approx 1$ and $\beta_{i} \ll 1$; where $\mathcal{M}_{A, i}$ is the initial value of the Alfvénic Mach number (the rms velocity of the flow divided by the Alfvén velocity) and $\beta_{i}=\left(P_{g} / P_{m}\right)_{i}$ is the initial ratio of gas pressure to magnetic pressure. Notice that the magnetic energy grows considerably in the beginning of the super-Alfvénic runs, and therefore the value of $\mathcal{M}_{A}$ can quickly decrease by a factor of two and the value of $\beta$ by a factor of four.

Both models $\mathrm{A}$ and $\mathrm{B}$ are divided in two subgroups: decaying runs (simply $\mathrm{A}$ and $\mathrm{B}$ ), and randomly driven runs ( $\mathrm{Ad}$ and $\mathrm{Bd}$ ). Models of the same type can also differ by the initial rms Mach number of the flow (the rms velocity of the flow divided by the speed of sound), or by the inclusion or not of ambipolar diffusion. A list of the models is given in Table 1, together with the initial values of their parameters.

In all experiments the initial density is uniform, and the initial velocity is random. We generate the velocity field in Fourier space, and we give power, with a normal distribution, only to the Fourier components in the shell of wave-numbers $1 \leq k L / 2 \pi \leq 2$. We perform a Helmholtz decomposition, and use only the solenoidal component of the initial velocity. The initial magnetic field is uniform, and is oriented parallel to the $z$ axis: $\mathbf{B}=B_{0} \hat{\mathbf{z}}$.

\section{Results}

In this section some properties of the models that may be related to observational quantities are discussed. The initial values of some physical parameters of these models are listed in Table 2. There are only two numerical parameters in the models: $\mathcal{M}$ and $\mathcal{M}_{A}$. In order to rescale the models to physical units, we use the following empirical Larson's relations (Larson 1981):

$$
\mathcal{M}=4.0\left(\frac{L}{1 p c}\right)^{0.5}
$$

where a temperature $T=10 \mathrm{~K}$ is assumed, and

$$
\langle n\rangle=2.0 \times 10^{3}\left(\frac{L}{1 p c}\right)^{-1}
$$

that is equivalent to a constant mean surface density. With these two relations, the value of $\mathcal{M}$ determines the value of $L$, the linear size of the model cloud, and of $\langle n\rangle$, the average number density. The physical unit of velocity in the code is the isothermal speed of sound, $C_{s}$, and the physical unit of the magnetic field is $C_{s}(4 \pi\langle\rho\rangle)^{\frac{1}{2}}(\mathrm{cgs})$.

\subsection{Dissipation of supersonic motions}

Molecular cloud lifetimes are estimated to be of the order of a few dynamical times, while supersonic motions should dissipate their kinetic energy in shocks in about one dynamical time. One of the original motivations for models of magnetized clouds with equipartition of kinetic, magnetic and gravitational energies was the belief that MHD waves decay on a longer time-scale than random super-sonic and super-Alfvénic flows, and therefore could offer an explanation for the long lifetime of molecular clouds. Zweibel \& Josafatsson (1983) and Elmegreen (1985), noticed that decay of MHD waves can be rather short, but their studies are based on the ambipolar diffusion dissipation. Ambipolar diffusion is in fact believed to be the most important dissipation mechanism in magnetized clouds (McKee et al., 1993).

We have run decaying experiments both with and without ambipolar diffusion, from which we can see that the inclusion of ambipolar diffusion does not change very much the decay time-scale. In our decaying equipartition models, the most important energy dissipation occurs because motions along the magnetic field lines produce strong shocks, just as in super-Alfvénic models. This was found also by Gammie and Ostriker (1996). The ratio of the decay time to the instantaneous dynamical time is plotted in Fig. 1 for models without ambipolar diffusion, and in Fig. 2 for similar models, but with the inclusion of ambipolar diffusion. Decay times of kinetic, magnetic and total energies are plotted versus the time in units of the initial dynamical time, $\tau_{d y n}=L / \sigma_{v}$. After a short initial transient (of the order of $0.5 \tau_{d y n}$ because the initial velocity field has power up to $k L / 2 \pi=2$ ), the decay time is in all cases comparable to the instantaneous dynamical time. Thus, the strength of the magnetic field, and also the importance of ambipolar diffusion, do not significantly affect the time-scale of decay of the flow.

A simple interpretation of the insensitivity of the level of dissipation to details of the dissipation mechanism is that it is related to the origin of the scaling behavior of turbulent flows. We observe a similar behavior in our super-sonic experiments as in experi- 
ments with sub-sonic turbulence; a power law energy power spectrum is established already after a fraction of a dynamical time. The scaling is such that the decay of energy does not depend on the particular dissipation process that occurs on the small viscous and resistive scales, but is rather determined by the large scale kinematics, characterized by the dynamical time-scale.

It may be concluded that equipartition models do not offer any advantage as far as the energy decay is concerned, compared with super-Alfvénic models. Mac Low (1998) and Stone et al. (1998) have recently shown that the decay time in equipartition models can be even shorter than in super-Alfvénic models. The problem of the lifetime of molecular clouds is not to be solved by the intervention of a strong magnetic field, but more likely by continuous driving of the turbulence. Cascading energy from distant supernova activity is a likely source of galactic turbulence (Korpi et al., 1998b; Korpi et al., 1998a; Korpi et al., 1999). Also, bipolar outflows from stars formed inside molecular clouds can provide significant amounts of energy (Reipurth, Bally \& Devine, 1997). Moreover, cloud life times are not very long (Blitz \& Shu 1980), and could perhaps even be comparable with their dynamical time (Elmegreen \& Efremov, 1998).

In this connection it should be pointed out that, even though energy sources on the average replenish turbulence in clouds, the driving may be intermittent in time as well as in space, and turbulence in individual clouds may thus, at times, be decaying.

\subsection{Probability Distribution of Magnetic Field Strength}

The main difference between the super-Alfvénic model and the equipartition model is the fact that in the latter the kinetic energy is not sufficiently large to compress the magnetic field significantly, while in the former very strong density enhancements produce large local enhancement of the magnetic field strength. Moreover, expansions generate large voids with magnetic field strength much smaller than the mean field in the super-Alfvénic model, but not in the equipartition model.

As a result, runs of type A produce very intermittent probability distributions of the field strength, with a roughly exponential tail. Models of type B, instead, are characterized by approximately Gaussian probability distributions of the field strength. In
Fig. 3, the field strength distributions from the last snapshots of model Ad2 (thick line) and model Bd1 (thin line) are plotted. In model $\mathrm{Ad} 2,\langle B\rangle=4.5 \mu \mathrm{G}$, but values of field strength up to $100 \mu \mathrm{G}$ are reached in high density regions. The values of $B$ span almost four orders of magnitude, $0.03 \mu \mathrm{G}<B<100 \mu \mathrm{G}$, in model Ad2, while they span only one order of magnitude in model $\mathrm{Bd} 1,4.5 \mu \mathrm{G}<B<46 \mu \mathrm{G}$.

In the super-Alfvénic model most of the mass concentrates in regions of large gas density (see below), where the field strength is also large. Because of this, approximately $0.5 \%$ of the total mass of the system contains a field 10 times stronger than the mean value. For example, the last snapshot of model Ad2 corresponds to molecular clouds on the scale of $15 \mathrm{pc}$, with $\langle n\rangle=133 \mathrm{H}_{2} \mathrm{~cm}^{-3}$. It may be taken, therefore, as a model for a molecular cloud of about $2 \times 10^{4} \mathrm{M}_{\odot}$. Since $\langle B\rangle=4.5 \mu \mathrm{G}$, one clump of about $100 \mathrm{M}_{\odot}$ can be found in the cloud, with $B \sim 45 \mu \mathrm{G}$, according to model Ad2.

The examples show that the formation of cores with rather large field strength is predicted in the super-Alfvénic model, even if the mean magnetic field strength in the cloud is quite small, and comparable with the mean galactic field strength. As a result, Zeeman splitting observations should not easily detect the magnetic field in clouds, apart from inside particularly favorable high density clumps. Conversely, if the magnetic field is detected in a cloud core, it is likely that the mean field in the cloud is much smaller than the estimated field strength in that core, if the cloud dynamics is described by a random super-sonic and super-Alfvénic flow, as in model Ad2.

\subsection{Probability Distribution of Gas Density: Stellar Extinction}

In a highly radiative gas, the most important effect of supersonic random flows is the fragmentation of the medium into filaments and clumps with very large density contrast. Supersonic turbulence can indeed be the reason for the observed fragmentation of molecular clouds (eg Padoan \& Nordlund 1998). It is interesting therefore to quantify the statistics of the density distribution that arise from supersonic turbulence. Vázquez-Semadeni (1994) found that the density distribution in his two dimensional numerical simulations was consistent with a Log-Normal. Padoan, Nordlund \& Jones (1997) also found density distributions consistent with a Log-Normal in their three dimensional numerical simulations, while Scalo 
et al. (1998) studied the effect of a polytropic equation of state on the density distribution. Nordlund \& Padoan (1998) used a new set of numerical experiments to show that even polytropic flows produce a density distribution similar to the Log-Normal, but slightly skewed. They also argue that such a distribution is due to the fact that the dynamics does not depend on the mean density.

Observationally it is difficult to measure the gas volume density in molecular clouds, but stellar extinction measurements can provide information about the projected gas density in clouds. Lada et al. (1994) suggested the use of stellar extinction measurements as a test for models of the structure of molecular clouds. Padoan, Jones, \& Nordlund (1997) have shown that near-infrared stellar extinction measurements can be used to infer the three dimensional probability distribution of the gas density in dark clouds. They have shown that there is qualitative and quantitative agreement between the inferred properties of the three dimensional density distribution in the dark cloud IC5146 (Lada et al. 1994), and the properties of the three dimensional density distribution in their experiments of random supersonic flows.

The comparison between numerical models and observational data is based on the plot of the dispersion of the extinction measurements in cells, versus the mean extinction in the same cells (Lada et al. 1994). In Padoan, Jones \& Nordlund (1997), the theoretical plots are calculated starting from random density fields of given statistics and power spectra. Here we generate the same plot, but calculated directly from the density field of the experiments. A random distribution of stars is generated and it is assumed that most stars are in the background (that is the model cloud is between the stars and the observer). The visual extinction is proportional to the column density of the cloud sampled by the line-of-sight to any given star:

$$
A_{V} / \operatorname{mag}=\frac{N\left(H+H_{2}\right)}{2 \times 10^{21} \mathrm{~cm}^{-2}}
$$

(Bohlin et al. 1978) . A uniform grid is used to subdivide the whole area into cells, each containing an average number of five stars, as in Lada et al. (1994). The mean extinction $A_{V}$ and its dispersion $\sigma\left(A_{V}\right)$ are measured inside each cell, and $\sigma\left(A_{V}\right)$ is plotted against $A_{V}$.

We have performed this test with all our models. In Fig. 1 we show the results for the models Ad2 and Bd1. These two models both have very large rms Mach number and differ from each other because model Ad2 is super-Alfvénic, while model Bd1 has approximate equipartition of magnetic and kinetic energy. We use the last snapshots from the two runs, where the values of Mach number and Alfvénic Mach number are $\mathcal{M} \approx 15$ and $\mathcal{M}_{A} \approx 5$ respectively, for model Ad2, and $\mathcal{M} \approx 12$ and $\mathcal{M}_{A} \approx 1$ for model Bd1. According to the Larson's relations we use in the present work to rescale our numerical models to physical units (Eqs. (7) and (8)), these snapshots correspond to molecular clouds on the scale of $14 \mathrm{pc}$ $(\mathrm{Ad} 2)$ and $9 \mathrm{pc}(\mathrm{Bd} 1)$. Using the data by Lada et al. (1994) and by Dobashi et al. (1992), one may infer an rms Mach number $\mathcal{M} \approx 10$ for the dark cloud IC5146, comparable to the Mach number values in the simulations. However, the particular scale is not important as for the mean or total column density of model or real clouds. This is because the Larson's relations are such that the column density of clouds is independent of size. The scale is instead important for determining the slope of the upper envelope of the $A_{V}-\sigma\left(A_{V}\right)$ plot. As shown in Padoan, Jones \& Nordlund (1997), that slope is roughly proportional to the standard deviation of the three dimensional density distribution, which is also proportional to the Mach number of the flow. So the slope is expected to be larger on larger scales (higher rms Mach number).

It is clear for Fig. 1 that the super-Alfvénic model reproduces very well the observed plot, while the equipartition model (Bd4) does not. Although model $\mathrm{Bd} 4$ has an rms Mach number comparable to the one in the cloud IC5146, the values of $A_{V}$ and $\sigma\left(A_{V}\right)$ in the model span a much smaller range than in the observations. This is also illustrated by the histogram of extinction (column density), shown on the left panels of Fig. 4 The equipartition model behaves like an elastic medium in the directions perpendicular to the magnetic field lines, and this reduces very much its ability to generate a sufficiently complex density field. In particular, while compressions on all three dimensions can generate a topology with large holes and adjacent lines-of-sights with very large fluctuations in extinction (model Ad2), compressions in only one dimension (model Bd1) can do that only to a smaller extent. 


\subsection{Synthetic spectra: line width versus in- tegrated temperature}

Most of the data from dark clouds consist of molecular emission line observations. The intensity of a particular emission line depends on many factors such as gas density, kinetic temperature, radiation field, and chemical abundances. Moreover, the line profiles are strongly dependent on the gas kinematics, but in a rather complex way, because only radial velocities are available, and both line widths and intensities are quantities projected along the line-ofsight. It is very difficult to extract reliable information about the three dimensional structure and kinematics of molecular clouds directly from observational data, and making use of simple models assuming local thermodynamic equilibrium (LTE), smooth velocity fields, and oversimplified density distribution. Part of the problem is due to the complexity of the cloud structure and of the internal random motions in clouds.

Padoan et al. (1998b) have recently calculated maps of synthetic molecular spectra that may be used to infer intrinsic properties of molecular clouds by comparison with observed spectra. Details of the method can be found in Padoan et al. (1998b). Here we simply remark that the synthetic spectra are obtained with a non-LTE Monte Carlo radiative transfer code (Juvela 1997), and the radiative transfer calculations are performed on a cloud model that is similar to the ones used in present work. Images of synthetic molecular maps of different transitions and molecules are shown in Padoan et al. (1998b), together with statistics of the spectral line profiles. In that work, a super-Alfvénic flow was used, and was shown to reproduce observed properties of clouds (see also Padoan et al., 1998a). In the present work we are interested also in the comparison between the superAlfvénic and equipartition models, and we have therefore solved the radiative transfer problem using cloud models Ad2 and Bd4 (the radiative transfer calculations have been kindly provided by Mika Juvela). Notice that although the initial Mach number of model $\mathrm{Ad} 2$ is almost 30, it later decreases (due to its initial conditions and not strong enough random driving), to a value comparable to the one in model $\mathrm{Bd} 4$. We use the last snapshots from each model, which correspond to the physical regime in typical molecular clouds on the scale of about $10-15 \mathrm{pc}$.

Comparisons of molecular spectra between the two models have shown that while the two cases are rather similar in many of their observable properties, they are very different in the relation between $\mathrm{J}=1 \rightarrow 0{ }^{13} \mathrm{CO}$ line width and $\mathrm{J}=1 \rightarrow 0{ }^{13} \mathrm{CO}$ integrated antenna temperature. The integrated antenna temperature is roughly proportional to the gas column density (cf. Dickman 1978, and Padoan et al., 1999a, for a critical discussion based on non-LTE radiative transfer), and its histogram is shown in Fig. 年. The equipartition model has a narrower distribution than the super-Alfvénic model, consistent with the discussion of dust extinction in the previous subsection. The histogram of line width is also narrower for model Bd4 that for model Ad2 (not shown), although the spectra averaged over all synthetic maps are almost identical (same line width). The relation between the line width and the integrated antenna temperature is also quite different in the two cases. The equipartition model shows almost no growth of line width with increasing $\mathrm{J}=1 \rightarrow 0{ }^{13} \mathrm{CO}$ antenna temperature, while in model $\mathrm{Ad} 2$ the growth is very significant, as shown in Fig.6.

Heyer, Carpenter \& Ladd (1996) find that in the star forming giant molecular clouds they observed the $\mathrm{J}=1 \rightarrow 0{ }^{12} \mathrm{CO}$ line width grows with $\mathrm{J}=1 \rightarrow 0{ }^{13} \mathrm{CO}$ integrated antenna temperature (roughly proportional to column density), and discuss the possibility of using this property to infer the dynamic importance of the magnetic field in the cloud motions. They argue that, if the motions observed in molecular clouds were Alfvén waves, like in theoretical models of dark clouds (for example in Carlberg \& Pudritz 1990), then the line width should decrease with column density. Based on this, they conclude that the motions in the clouds they observed are not consistent with Alfvén waves. We find that the $\mathrm{J}=1 \rightarrow 0{ }^{13} \mathrm{CO}$ line width in the equipartition model is in general almost independent of column density $\left(\mathrm{J}=1 \rightarrow 0{ }^{13} \mathrm{CO}\right.$ integrated antenna temperature), and in some cases even decreases with column density, as predicted by Heyer, Carpenter and Ladd (1996) for the $\mathrm{J}=1 \rightarrow 0{ }^{12} \mathrm{CO}$ line width. The equipartition model is therefore in conflict with the observations, while the super-Alfvénic model shows a relation between line width and column density that is similar to the observational result.

In order to better constrain the importance of the magnetic field in the dynamics of molecular cloud internal motions, it would be useful to measure line width versus integrated temperature in many different clouds. We expect that the magnetic field could 
play an important role in some cores with large field strength, and this could appear in the relation between $\mathrm{J}=1 \rightarrow 0{ }^{13} \mathrm{CO}$ line width and column density. Cores with strong magnetic field (predicted in the present super-Alfvénic scenario for molecular cloud dynamics) have already been identified by $\mathrm{OH}$ Zeeman splitting measurements, and should be the target of such a study.

\subsection{The $B-n$ relation}

As discussed above, the equipartition model generates a smaller density contrast than the superAlfvénic model, and also generates much smaller fluctuations in the magnetic field strength. The relation between field strength and gas density ( $B-n$ relation) is therefore expected to be rather different in the two models. The $B-n$ relation in the models may be compared with observational results.

In regions of maser emission, at densities of about $n=10^{7} \mathrm{~cm}^{-3}$, a field strength of the order of $B=$ $10^{3}-10^{4} \mu G$ is observed, while in regions of molecular emission, with approximately $n=10^{2}-10^{3} \mathrm{~cm}^{-3}$, the field is found to be of the order of $B=10 \mu G$ (eg Myers \& Goodman 1988). A relation of the type $B \propto n^{0.3-0.6}$ may be deduced from the observations (eg Troland, Crutcher \& Kazès 1986; Heiles 1987; Dudorov 1991), but it is quite uncertain, especially in the light of the above discussion about the intermittency of the distribution of the magnetic energy.

We have updated the $B-n$ relation by Troland \& Heiles (1986) with more recent measurements: the HI Zeeman observations by Verschuur (1995); the $\mathrm{OH}$ and CN Zeeman splitting measurements by Crutcher et al. $(1993,1996,1998)$; the $\mathrm{OH}$ maser measurements by Johnston et al. (1989); the $\mathrm{H}_{2} \mathrm{O}$ maser measurements by Fiebig \& Gusten (1989).

In Fig. [, observational detections of the fieldstrength and upper limits are plotted versus the estimated value of the gas density. Although most of the attempts to detect the Zeeman effect result in non-detections, and therefore in upper limits to the field-strength, there are some detections of $B$ also in the range of density found in molecular clouds. $B$ is of course detected preferentially in the regions where it is particularly strong, which are hardly representative of the typical physical conditions inside molecular clouds. Fig. 7 shows a clear power-law relation between $B$ and $n$, defined by the regions with strongest magnetic field. At the same time, there are many es- timated upper limits (over 100 listed in publications and only summarized in Fig. 7) that very likely probe many regions with values of $B$ weaker than the ones detected in regions of similar densities. The observations therefore clearly indicate that the $B-n$ relation has a large scatter, although a power-law upper envelope is well defined over approximately 10 orders of magnitude in gas density and 5 orders of magnitude in field-strength. The upper envelope is roughly

$$
B \approx 100 \mu G\left(\frac{n}{10^{3} c^{-3}}\right)^{0.4}
$$

and the scatter at $n=10^{3} \mathrm{~cm}^{-3}$ is at least two orders of magnitude, in the approximate range $1-100 \mu \mathrm{G}$.

A super-Alfvénic flow (eg model Ad2) naturally develops a $B-n$ relation with a power law upper envelope and a large scatter, and also provides a large density contrast at the same time. The $B-n$ relation is initially almost linear (see below for an explanation), and flattens with time. The upper envelope of the relation also flattens with time, and after about one dynamical time it stabilizes to $B \propto n^{0.4}$, as in the observations. In Fig. 7 contour lines obtained from the $B-n$ scatter plot in model Ad2 (thick lines) are shown, together with the observational data. Model Ad2 corresponds to typical molecular clouds on the scale of 20 pc (rms Mach number $\approx 20)$. The particular snapshot used in Fig. 7 has $\mathcal{M}=15$ and $\langle B\rangle=4.5 \mu \mathrm{G}$. Although the mean field strength is so low (of the order of the mean galactic field), the fluctuations of field strength are every large $(0.03<B<100 \mu \mathrm{G})$, and the largest values of the magnetic field strength (upper envelope) at each density are very close to the observational values. In model $\mathrm{Ad} 2$ there is a clear power-law upper envelope over about 4 orders of magnitude in density and almost 2 in $B$, which matches the observations nicely. The scatter in the $B-n$ relation of model Ad2 is also consistent with the observations. In order to achieve the densest values of field strength estimated in molecular clouds using CN Zeeman splitting and masers, higher numerical resolution would be necessary, or perhaps self-gravity should be included in the calculations.

The result for an equipartition model (Bd1) is also overplotted in Fig. 1 (thin lines). The model is inconsistent with the observations in two ways: i) the ranges of density and field strength values are too small (see the two subsections above) and ii) neither the upper envelope nor the mean field strength at each density define a $B-n$ relation similar to the observa- 
tional one. In particular, most observational upper limits on the field strength, and a few detections, are completely inconsistent with the equipartition model.

While super-sonic and super-Alfvénic turbulence naturally explains the results of Zeeman splitting measurements, including the $B-n$ relation and its scatter, such results are not easily explained by the alternative equipartition model.

The lack of correlation between $B$ and $n$ in model $\mathrm{Bd} 1$ is due to the fact that most of the density enhancement occurs by convergent flows along the field lines, and these motions do not affect the magnetic field.

One could possibly argue that the lack of correlation of $B$ and $n$ in model $\mathrm{Bd} 1$ is consistent with the majority of observations, because often no field is detected, and hence nothing can be said about the correlation. However, as further discussed in the next Section, the upper limits of the non-detections speak against this interpretation. Also, the cases where a field is detected would then have to be explained with ad hoc arguments, rather than as a natural part of a statistical distribution.

\subsection{Cloud and flow topology}

An understanding of the spatial structure of the density field in dark clouds is very important for a correct interpretation of observational data, and for the formulation of a number of physical models. In this subsection we provide our interpretation of the dynamical phenomena occurring in the simulations, based on extensive three dimensional browsing of the data, and illustrated here by images of the density field and plots of statistical correlations of the velocity and magnetic fields.

The snapshots in Fig. 8 and Fig. 9 give an idea of the dimensionality of the structures in the density field. The topology of the density field in experiment Ad2 (Fig. 8) has a clear evolution in time. In the very beginning, until $t \approx 0.6 t_{d y n, 0}$, the density grows predominantly in sheets. These are the fronts of blobs of coherent motion, advancing at supersonic velocity. Later, these fronts start to intersect each other, and the density increases especially in filaments (at the intersections of fronts). The evolution continues with the intersection of filaments into knots, at $t \approx 1.5 t_{d y n, 0}$. The fully developed topology, at $t=2.0 t_{d y n}$, is characterized by both filaments and knots (cores). Also in experiment Bd1 (Fig. 9) the density grows initially in sheets, but the transition from sheets to filaments is mainly due to motions along field lines, rather than to intersection of sheets. In the equipartition model Bd1, in fact, density enhancements cannot move freely in the three space directions, but only along magnetic field lines. It is therefore typical that density enhancements such as sheets are torn apart, by motions along field lines, into filaments approximately aligned with the magnetic field. The approximate alignment of the filaments is visible in the left panel of Fig. 9. Later in time, the density structure becomes more complex (right panel of Fig. 9), but still shows some evidence of the alignment of the density filaments.

The evolution of the magnitudes of the mass density and the magnetic field may be discussed with reference to Lagrangian version of the continuity equation,

$$
\frac{\mathrm{D} \ln \rho}{\mathrm{D} t}=-\nabla \cdot \mathbf{v}
$$

and the scalar induction equation

$$
\frac{\mathrm{D} \ln |B|}{\mathrm{D} t}=-\nabla \cdot \mathbf{v}_{\perp}+\hat{B} \cdot(\hat{B} \cdot \nabla) \mathbf{v}_{\perp},
$$

where $\mathbf{v}_{\perp}$. is the velocity perpendicular to the magnetic field, and $-\nabla \cdot \mathbf{v}_{\perp}$ usually is the dominant term on the RHS.

Although $-\nabla \cdot \mathbf{v}$ vanishes for the solenoidal initial condition, the supersonic motions rapidly lead to the formation of shock fronts, where the local value of $-\nabla \cdot \mathbf{v}$ is large and positive because of the discontinuity in the velocity perpendicular to the shock.

The initially homogeneous magnetic field is carried along by the perpendicular components of the velocity field, and is hence also collected into sheets, except at those rare locations where the initial field happens to be strictly parallel to the velocity field. This explains why sheets initially form in both mass density and magnetic flux density, and why the $B-n$ relation initially has an exponent close to unity.

Note that the usual argument for a slope of $2 / 3$, that applies to isotropic and non-shocking compressible motion does not apply here, because of the development of discontinuities. In term of Eqs. 11 and 12, the $2 / 3$ follows if $-\nabla \cdot \mathbf{v}_{\perp}$ typically picks up two of three statistically equivalent contributions to $-\nabla \cdot \mathbf{v}$. However, at a shock, the divergence is dominated by the derivative in one particular direction; the one perpendicular to the shock front. The magnetic field that is swept into the discontinuity quickly becomes almost 
parallel to the shock front, because the component in the plane of the shock grows exponentially with time. Thus, as long as the topology is dominated by sheets, the mass density and the magnetic flux grow more or less in unison in the sheets, corresponding to an exponent in the $B-n$ relation close to unity.

In the subsequent evolution, there are effects that tend to reduce the exponent in the $B-n$ relation. First, the non-linear evolution of the initially solenoidal velocity field also leads to the development of regions of space with a positive divergence, in which both the mass density and the magnetic flux density decline. In these regions, there is no particular dominance of the cross-field divergence, and thus the three-dimensional divergence picks up an additional contribution relative to the two-dimensional divergence, consistent with the classical $2 / 3$ argument outlined above.

In experiment B1 the motions are mainly Alfvén waves, and therefore the velocity is predominantly perpendicular to the direction of the magnetic field. This is illustrated in Fig. 10, where we have plotted the histogram of $\cos (\alpha)$, where $\alpha$ is the angle between $\mathbf{v}$ and $\mathbf{B}$. Note that, even though motions across the field lines are the most common ones, it is the less frequent motions along the field lines that dominate the dissipation. The motions across field lines are subject to magnetic restoring forces, and do not lead to substantial density enhancements.

In experiment A1, the magnetic field is advected by the flow, and the stretching of field lines instead produces some alignment between $\mathbf{v}$ and $\mathbf{B}$, already before one dynamical time has passed, as illustrated in Fig. 10.

Alignment between $\mathbf{B}$ and $\mathbf{v}$ may be caused by two, complementary effects: 1) Dynamical alignment is expected when the magnetic energy approaches and exceeds the kinetic energy; the Lorentz force then forces the flow to be predominantly along the magnetic field lines. 2) Kinematic alignment occurs when a spatially non-uniform velocity field causes stretching of magnetic field lines, and hence a correlation of $\mathbf{B}$ and $\mathbf{v}$. Pure shear, for example, tends to align an embedded magnetic field with the direction of the flow.

Motions that are aligned with the magnetic field affect the mass density without affecting the magnetic flux density. In particular, the non-linear concentration into first sheets and then filaments due to the interaction of shock fronts continues into the formation of knots in the density field, by the convergence of matter flowing along filaments. There is no corresponding process available to a divergence free vector field such as the magnetic field; once the field has concentrated into filaments, it cannot concentrate further; the magnetic field in a filament is insensitive to flow along the filament.

In the same way that converging flows along the magnetic field may lead to extreme concentrations of mass, those regions where the flow is diverging along magnetic field lines may lead to extreme rarefactions of mass, without affecting the magnetic flux density. In $B-n$ scatter plots, this corresponds to the development of more extreme excursions of the mass, relative to those of the magnetic flux density, and hence a flattening of the $B-n$ relation with time.

In model A1, dynamical alignment is at most significant in the few cores that develop a strong (subAlfvénic) magnetic field in the early evolution of the experiment. In scatter plots of $B$ against $n$ most contributions come from regions where dynamical alignment is unimportant. We thus conclude that the evolution of the $B-n$ relation in model $\mathrm{A}$, towards a smaller exponent with time, is caused by the kinematic alignment of $\mathbf{B}$ and $\mathbf{v}$.

\section{Discussion}

It is difficult to get an objective view on the magnetic field strength in dark clouds from the literature. The reasons are the following:

- Negative results from observational programs in which detections have been reported are likely to remain unpublished.

- The positions searched for Zeeman splitting never represent a statistically meaningful sample. Favorable regions are always selected, because the observations are very time consuming.

- The total number of regions in dark clouds, for which $\mathrm{OH}$ Zeeman observations are published, is still small.

Although all Zeeman splitting measurements are biased towards regions where a strong field is expected (high density cores), in many cases the field is not detected, and upper limits are quite stringent. As an example, Crutcher et al. (1993) selected four cores in the Taurus dark cloud complex, two in the Libra complex, two in $\rho \mathrm{Oph}$, one in the Orion molecular ridge, 
one position in L889, and the core of B1 (Barnard 1), in the Perseus region. The only certain detection is in the cloud B1. For the other regions, the weighted average value of the field is $+2.7 \pm 1.5 \mu G$ in Taurus, $-2.1 \pm 2.8 \mu G$ in Libra, $+6.8 \pm 2.5 \mu G$ in $\rho \mathrm{Oph}$, $-0.6 \pm 2.1 \mu G$ in $\mathrm{L} 889$, and $-4.7 \pm 3.5 \mu G$ in L1647. CN Zeeman splitting measurements have also provided rather low upper limits to the field strength, compared with estimated equipartition values (Crutcher et al. 1996), and also magnetic field detections well below equipartition (Crutcher et al. 1998). A rather low average field of $+9 \mu G$ was also found in Cas A by Heiles and Stevens (1986).

Orion A and Orion B are instead two examples of detections of intense magnetic field. OH Zeeman splitting in Orion A revealed a magnetic field strength of $B=-125 \mu G$ (Troland, Crutcher, \& Kazès 1986), and $B=+38 \mu G$ toward Orion B (Crutcher \& Kazès 1983).

The $\mathrm{OH}$ Zeeman splitting should probe regions with $n=10^{3}-10^{4} \mathrm{~cm}^{-3}$, while the CN Zeeman splitting, should probe regions of $n \approx 10^{6} \mathrm{~cm}^{-3}$.

In our model $\mathrm{Ad} 2$, the mean field strength is $B=$ $4.5 \mu \mathrm{G}$, and the mean density $\langle n\rangle=1.3 \times 10^{2} \mathrm{~cm}^{-3}$; $20 \%$ of the total mass is in dense clumps, with density ten times larger than the mean, $n \approx 3 \times 10^{3} \mathrm{~cm}^{-3}$, and field strength five times, or more, larger than the mean, $B \geq 25 \mu \mathrm{G}$. Therefore, even if field strengths of about $40 \mu G$ are detected sometimes in dense cores, the mean Alfvén velocity in the molecular cloud may be just comparable to the sound speed, $\left\langle v_{A}^{2}\right\rangle^{1 / 2} \approx C_{S}$.

It may be concluded that model $\operatorname{Ad} 2$ is consistent with the observational estimates of magnetic field strength in dark clouds. The particular values of the magnetic field used in the comparison here should not be taken too literally; the small scale field strengths could be larger than estimated by the Zeeman effect if the magnetic field is tangled (but notice that in Fig. 0 the observational measurements have been multiplied by a factor of two, to account for the random orientation of the magnetic field lines relative to the lineof-sight).

It is difficult to envisage how the measurements could be consistent with equipartition models such as $\mathrm{Bd} 1$ or Bd4, however, since the kinetic energy does not exceed the magnetic energy. As demonstrated by Galsgaard \& Nordlund (1996), a magnetically dominated plasma is able to quickly dissipate structural complexity, independent of the value of the resistivity.
Thus, a sub-Alfvénic field could not remain strongly tangled, and hence could not avoid detection. Using the same argument, we expect those cores where a strong (sub-Alfvénic) field has indeed been observed to have a relatively simple magnetic field structure.

Model Ad2 can be used for the description of a typical molecular cloud with linear size of about $15 \mathrm{pc}$ and $\langle n\rangle=130 \mathrm{~cm}^{-3}$. At the time $t=1.0 t_{d y n}$, the relation $B-n$ is then:

$$
B \approx 4.5 \mu G\left(\frac{n}{100 \mathrm{~cm}^{-3}}\right)^{0.7}
$$

Since the exponent is $>0.5$, most of the dense cores are found to have magnetic pressure larger than thermal pressure, at early times. The lowering with time of the exponent in the $B-n$ power law means that later on, the dominance of the magnetic field in dense cores tends to be reduced. Although the flow is random and approximately isotropic, the kinematic alignment of $\mathbf{B}$ with $\mathbf{v}$ makes dense cores accrete mass along $\mathbf{B}$ at an increased rate. Therefore, the accretion of mass around dense cores, embedded in a random flow with $\beta \approx 1$, is such that, while magnetic pressure becomes dominant over thermal pressure during the initial phase of turbulent fragmentation, later on, in some cores, magnetic pressure can decreases to the level of thermal pressure, even in the absence of gravity, and on a time scale that is competitive with ambipolar diffusion. This mechanism could be relevant for the process of star formation.

A central point that we want to stress in this paper is that the fact of finding some cloud cores, with sub-Alfvénic velocity dispersions and with magnetic pressure larger than thermal pressure, does not necessarily mean that the dynamics of molecular clouds is dominated by MHD waves; those cores may be formed, in a few million years, in a super-sonic and super-Alfvénic flow, only marginally affected by the magnetic field (model Ad2). The dynamics becomes strongly affected by the magnetic field only in some very dense regions, on small scales, and preferentially during the first dynamical time.

We stress that a key theoretical ingredient to the interpretation of $\mathrm{OH}$ Zeeman splitting data is the $B$ $n$ relation. The fact that the exponent of the relation is $>0.5$ for more than one dynamical time is the reason why sub-Alfvénic cores can be found in the experiment.

An interesting question is whether the present results, that indicate that kinetic energy dominates 
over magnetic energy on the scale of cold clouds, could still be consistent with the well established rough equipartition of kinetic and magnetic energies on larger scales, e.g. comparable with the thickness of the galactic disc (Boulares \& Cox, 1990). Here, we can only offer some preliminary remarks; a more quantitative answer requires larger scale and higher resolution numerical simulations, that go beyond our current numerical capabilities.

However, we have made a rather interesting observation already in the current simulations. It turns out that, while the power spectra of velocity and magnetic field strength are nearly parallel, the spectra of kinetic and magnetic energy are not. Because of the intermittency of mass density $\rho$ that develops in these supersonic flows, the power spectrum of $\rho^{\frac{1}{2}} \mathbf{V}$ need not be parallel to the power spectrum of $\mathbf{v}$. In our super-Alfvénic experiments there is a difference of slope between the power spectra of magnetic and kinetic energy, amounting to about 0.5 , with the kinetic energy spectrum being more shallow.

Such a difference in slope opens up the interesting possibility that, over the $2-3$ orders of magnitude that separate cold clouds from large scale disc structures, a difference of about an order of magnitude develops between the kinetic and magnetic energy densities. This is roughly consistent with the differences between kinetic and magnetic energy adopted in our super-Alfvénic models. More firm conclusion on this issue must await models with higher Mach number and higher numerical resolution.

\section{Conclusions}

In this work we have shown that:

- Both super-Alfvénic and equipartition random super-sonic flows, are characterized by a decay time-scale approximately equal to one dynamical time. One of the original motivations for the theoretical models of magnetized clouds with rough equipartition between kinetic, gravitational, and magnetic energies was the belief that in such models the decay time-scale could be significantly longer than one dynamical time. Since this is now known to be incorrect (see also MacLow et al. 1998 and Stone, Ostriker \& Gammie 1998), equipartition models of molecular clouds have lost their original motivation.

- Random super-sonic and super-Alfvénic mo- tions produce a very intermittent probability distribution of magnetic field strength, with an exponential tail. Cores with field strength several times in excess of the mean field can be formed.

- Random super-sonic and super-Alfvénic motions also produce a very intermittent probability distribution of gas density, in agreement with stellar extinction measurements, while equipartition models fail to reproduce the observational result.

- Synthetic spectra calculated from both the superAlfvénic and the equipartition models are used to study the correlation between line width and gas column density. The equipartition models fail again to reproduce qualitatively the growth of line width with column density, while the super-Alfvénic model shows a correlation similar to the observed one.

- Dense cores, with magnetic pressure larger than thermal pressure, and velocity dispersions smaller than $v_{A}$, are found as the result of the evolution of supersonic and super-Alfvénic flows.

- A power law statistical $B-n$ relation is generated by super-sonic and super-Alfvénic motions, but not by MHD waves in the equipartition model. The exponent of the relation is $>0.5$ for about one dynamical time, which allows for the existence of cores with $v_{A}>C_{S}$.

- The exponent in the $B-n$ relation of the superAlfvénic model decreases with time, because magnetic field lines are stretched and partially aligned with the flow. The statistical importance of the magnetic pressure in dense cores thus decreases with time, even in the absence of gravity and ambipolar diffusion. After about one dynamical time, the exponent of the upper envelope of the $B-n$ relation, is approximately equal to 0.4 , as in the observations.

- The scatter in the $B-n$ relation of the superAlfvénic model is also consistent with the Zeeman splitting measurements, while the scatter in the equipartition model is inconsistent with most Zeeman splitting upper limits on the field strength, and even with some field detections. 
In summary, even though the evidence presented in the present paper is far from complete, it appears that super-Alfvénic motions produces in a natural way the same type of statistical properties that characterize cold molecular clouds, while motions with equipartition of kinetic and magnetic energies have properties that are harder to reconcile with the observations.

This work has been partially supported by the Danish National Research Foundation through its establishment of the Theoretical Astrophysics Center.

We thank Enrique Vázquez-Semadeni for a very detailed and very useful (second opinion) referee report, and Prof. L. Mestel and Prof. E. Zweibel for their comments on the manuscript.

\section{References}

Arny, T. 1971, ApJ, 169, 289

Arons, J., Max, C. E. 1975, ApJ, 196, L77

Blitz, L., Shu, F. H. 1980, ApJ, 238, 148

Bohlin, R. C., Savage, B. D., Drake, J. F. 1978, ApJ, 224,132

Bonazzola, S., Falgarone, E., Heyvaerts, J., Perault, M., Puget, J. L. 1987, A\&A, 172, 293

Bonazzola, S., Perault, M., Puget, J. L., Heyvaerts, J., Falgarone, E., Panis, J. F. 1992, J. Fluid Mech., 245,1

Boulares, A., Cox, D. P. 1990, ApJ, 365, 544

Brandenburg, A., Zweibel, E. G. 1994, ApJ, 12, 279

Carlberg, R. G., Pudritz, R. E. 1990, MNRAS, 247, 353

Chandrasekhar, S. 1958, Proc. Roy. Soc., 246, 301

Crutcher, R. M. 1998, in J. Franco, A. Carramiñana (eds.), Interstellar Turbulence, Cambridge University Press

Crutcher, R. M., Kazès, I. 1983, A\&A, 125, L23

Crutcher, R. M., Troland, T. H., Goodman, A. A., Heiles, C., Kazès, I., Myers, P. C. 1993, ApJ, 407, 175

Crutcher, R. M., Troland, T. H., Lazareff, B., Kazès, I. 1996, ApJ, 456, 217

Dame, T. M., Elmegreen, B. G., Cohen, R. S., Thaddeus, P. 1986, ApJ, 305, 892

Dickman, R. L. 1978, ApJS, 37, 407

Dudorov, E. A. 1991, Sov. A., 35(4), 342

Elmegreen, B. G. 1985, ApJ, 299, 196
Elmegreen, B. G. 1997, ApJ, 477, 196

Elmegreen, B. G., Efremov, Y. 1998, in IAU Symposium 190, New Views of the Magellanic Clouds, 41

Elmegreen, B. G., Falgarone, E. 1996, ApJ, 471, 816

Elmegreen, B. G., Fiebig, D. 1993, A\&A, 270, 397

Falgarone, E., Pérault, M. 1987, in G. Morfil, M. Scholer (eds.), Physical Processes in Interstellar Clouds, Reidel, Dordrecht, 59

Falgarone, E., Puget, J. L. 1986, A\&A, 162, 235

Falgarone, E., Puget, J. L., Pérault, M. 1992, A\&A, 257,715

Fleck, R. C. J. 1981, ApJ, 246, L151

Galsgaard, K., Nordlund, A. 1996, Journal of Geophysical Research, 101(A6), 13445

Galsgaard, K., Nordlund, A. 1997, Journal of Geophysical Research, 102, 231

Gammie, C. F., Ostriker, E. C. 1996, ApJ, 466, 814

Goldsmith, P. F., Arquilla, R. 1985, ApJ, 297, 436

Heiles 1987, in D. J. Hollenbach, H. A. Thronson (eds.), Interstellar Processes, Reidel, 171

Heiles, C., Stevens, M. 1986, ApJ, 301, 331

Heyer, M. H., Carpenter, J. M., Ladd, E. F. 1996, ApJ, 463, 630

Houlahan, P., Scalo, J. 1992, ApJ, 393, 172

Hyman, J. 1979, in R. Vichnevetsky, R. S. Stepleman (eds.), Adv. in Comp. Meth. for PDE's-III, 313

Kimura, T., Tosa, M. 1993, ApJ, 406, 512

Kolmogorov, A. N. 1941, Dokl. Akad. Nauk. SSSR, 30,301

Korpi, M., Brandenburg, A., Shukurov, A., Tuominen, I. 1998a, in Proceedings of the 5th International Workshop "Planetary and Cosmic Dynamos", Vol. 42 of Studia Geoph. et Geod, Trest, Czech Republic,

Korpi, M., Brandenburg, A., Shukurov, A., Tuominen, I. 1998b, in J. Franco, A. Carramiñana (eds.), Interstellar Turbulence, Cambridge University Press, p. E16,

Korpi, M. J., Brandenburg, A., Shukurov, A., Tuominen, I., Nordlund, A., Stein, R. F. 1999, A Supernova Regulated ISM: Simulations of the Turbulent Multiphase Medium, ApJ, (submitted)

Lada, C. J., Lada, E. A., Clemens, D. P., Bally, J. 1994, ApJ, 429, 694

Langer, W. D., Velusamy, T., Kuiper, T. B. H., Levin, 
S., Olsen, E., Migenes, V. 1995, ApJ, 453, 293

Larson, R. B. 1981, MNRAS, 194, 809

Lee, S., Lele, S. K., Moin, P. 1991, Phys. Fluids A, 3, 657

Léorat, J., Passot, T., Pouquet, A. 1990, MNRAS, 243, 293

Leung, C. M., Kutner, M. L., Mead, K. N. 1982, ApJ, 262,583

Mac Low, M.-M. 1998, in J. Franco, A. Carramiñana (eds.), Interstellar Turbulence, Cambridge University Press

McKee, C., Zweibel, E., Goodman, A., Heiles, C. 1993, in E. H. Levy, J. I. Lunine (eds.), Protostars and Planets III, (University of Arizona Press, 327

McKee, C. F., E.G., Z. 1995, ApJ, 440, 686

Mestel, L. 1965, Q. Jl. R.A.S., 6, 161

Morris, M., Zuckerman, B., Turner, B. E., Palmer, P. 1974, ApJ, 192, L23

Mouschovias, T. C. 1976a, ApJ, 207, 141

Mouschovias, T. C. 1976b, ApJ, 206, 753

Mouschovias, T. C., Psaltis, D. 1995, ApJ, 444, L105

Myers, P. C. 1983, ApJ, 270, 105

Myers, P. C., Goodman, A. A. 1988, ApJ, 326, L27

Myers, P. C., Khersonsky, V. H. 1995, ApJ, 442, 186

Nordlund, Å., Galsgaard, K. 1997, A 3D MHD Code for Parallel Computers, Technical report, Astronomical Observatory, Copenhagen University

Nordlund, Å., Galsgaard, K., Stein, R. F. 1994, in R. J. Rutten, C. J. Schrijver (eds.), Solar Surface Magnetic Fields, Vol. 433, NATO ASI Series

Nordlund, Å., Padoan, P. 1998, in J. Franco, A. Carraminana (eds.), Interstellar Turbulence, Proceedings of the 2nd Guillermo Haro Conference, Cambridge University Press, E62

Nordlund, Å., Stein, R. F., Galsgaard, K. 1996, in J. Wazniewski (ed.), Proceedings from the PARA95 workshop, Vol. 1041 of Lecture Notes in Computer Science, Springer, p. 450

Norman, C., Silk, J. 1980, ApJ, 238, 158

Padoan, P. 1995, MNRAS, 277, 377

Padoan, P., Jones, B., Nordlund, Å. 1997a, ApJ, 474, 730

Padoan, P., Juvela, M., Bally, J., Nordlund, Å. 1998, ApJ, 504, 300

Padoan, P., Juvela, M., Bally, J., Nordlund, Å. 1999a, Super-Sonic Turbulence in The Perseus Molecular
Cloud, ApJ, (submitted)

Padoan, P., Juvela, M., Bally, J., Nordlund, Å. 1999b, A Theoretical Calibration of the ${ }^{13} \mathrm{CO}$ Column Density in Molecular Clouds, ApJ, (submitted)

Padoan, P., Nordlund, Å. 1998, in J. Franco, A. Carramiñana (eds.), Interstellar Turbulence, Cambridge University Press

Padoan, P., Nordlund, Å., Jones, B. 1997b, MNRAS, 288, 145

Padoan, P., Örnólfur E. Rögnvaldsson, Nordlund, Å. 1999b, ApJ, (in preparation)

Parker, D. A. 1973, MNRAS, 163, 41

Passot, T., Pouquet, A. 1987, J. Fluid Mech., 181, 441

Passot, T., Pouquet, A., Woodward, P. 1988, A\&A, 197, 228

Passot, T., Vázquez-Semadeni, E., A., P. 1995, ApJ, 455, 536

Porter, D. H., Pouquet, A., Woodward, P. 1992, Theoret. Comput. Fluid Dyn., 4, 13

Porter, D. H., Pouquet, A., Woodward, P. R. 1994, Phys. Fluids, 6, 2133

Quiroga, R. J. 1983, Ap. Sp. Sci., 93, 37

Reipurth, B., Bally, J., Devine, D. 1997, AJ, 114, 2708

Sanders, D. B., Scoville, N. Z., Solomon, P. M. 1985, ApJ, 289, 372

Scalo, J. M. 1985, in D. C. Black, M. S. Mathews (eds.), Protostars and Planets II, Tucson: University of Arizona Press, 201

Scalo, J. M., Pumphrey, W. A. 1982, ApJ, 258, L29

Scalo, J. M., Vázquez-Semadeni, E., Chappell, D., Passot, T. 1998, ApJ, 504, 835

Stein, R. F., Galsgaard, K., Nordlund, A. 1994, in J. D. B. et al. (ed.), Proc. of the Cornelius Lanczos International Centenary Conference, Society for Industrial and Applied Mathematics, Philadelphia, p. 440

Stenholm, L. G., Pudritz, R. E. 1993, ApJ, 416, 218

Stone, J. M., Ostriker, E. C., Gammie, C. F. 1998, ApJ, 508, L99

Strittmatter, P. A. 1966, MNRAS, 132, 359

Tagger, M., Falgarone, E., Shukurov, A. 1995, A\&A, 299, 940

Troland, T. H., Crutcher, R. M., Kazès, I. 1986, ApJ, 304, L57

Vázquez-Semadeni, E. 1994, ApJ, 423, 681 
Vázquez-Semadeni, E., Ballesteros-Paredes, J., Rodríguez, L. F. 1997a, ApJ, 474, 292

Vázquez-Semadeni, E., Passot, T., Pouquet, A. 1995, A\&A, 441, 702

Vázquez-Semadeni, E., Passot, T., Pouquet, A. 1997b, ApJ, 473, 881

Xie, T. 1997, ApJ, 475, L139

Zuckerman, B., Evans, N. J. 1974, ApJ, 192, L149

Zuckerman, B., Palmer, P. 1974, ARA\&A, 12, 279

Zweibel, E. G., Brandenburg, A. 1997, ApJ, 478, 563

Zweibel, E. G., Josafatsson, K. 1983, ApJ, 270, 511

This 2-column preprint was prepared with the AAS IATEX macros $\mathrm{v} 4.0$. 


\section{Figure and Table captions:}

Table 1: Initial parameters of the numerical experiments.

$\mathcal{M}_{A}$ : alfvénic rms Mach number;

$b_{0}$ : magnetic field strength parameter;

$\mathcal{M}$ : rms Mach number;

ambi: ambipolar diffusion parameter;

Table 2: Initial physical parameters of the models, obtained by scaling the numerical parameters of the experiments (Table 1) with the Larson's relations (Eqs. 7 and (8)).

Fig. 1: Time evolution of the ratio of the energy decaying time divided by the instantaneous dynamical time. Separate plots are for the total energy $E_{t o t}$, the magnetic energy $E_{m}$, and the kinetic energy $E_{k}$.

Fig. 2: Same as in Fig. 1, but for models with ambipolar diffusion.

Fig. 3: Distribution of magnetic field strength in the super-Alfvénic model Ad2 (thick line), and in the equipartition model Bd1 (thin lines). The dashed vertical lines mark the mean field strength in the two models.

Fig. 4: Histograms of extinction (left panels) and plots of dispersion of extinction in cells versus the mean cell extinction (right panels). The top panels show the result from the super-Alfvénic model Ad2, while the middle panels are from the equipartition model Bd1. The bottom panels are Lada et al. (1994) observational data for the cloud IC5146.

Fig. 5: Histograms of integrated antenna temperature of synthetic $\mathrm{J}=1 \rightarrow 0{ }^{13} \mathrm{CO}$ spectra, from the superAlfvénic model Ad2 (thick line) and for the equipartition model Bd1 (thin line). Model Ad2 shows a very intermittent approximately exponential distribution.

Fig. 6: $\mathrm{J}=1 \rightarrow 0{ }^{13} \mathrm{CO}$ equivalent width versus $\mathrm{J}=1 \rightarrow 0{ }^{13} \mathrm{CO}$ integrated antenna temperature, from the superAlfvénic model Ad2 (upper panel) and for the equipartition model Bd1 (lower panel).

Fig. 7: The $B-n$ relation: observations and theoretical models. The thick contour lines are from the the super-Alfvénic model Ad2, and the thin contour lines from the equipartition model Bd1.

Fig. 8: Three dimensional visualizations of the density field in the super-Alfvénic model Ad2, obtained by the superposition of voxel projection and density isosurfaces. The left panel corresponds to $t=0.5 t_{d y n, 0}$, and the right panel $t=1.5 t_{d y n, 0}$.

Fig. 9: Same as in Fig. 8 but for the equipartition model Bd1. The left panel corresponds to $t=1.3 t_{d y n, 0}$, and the right panel $t=2.8 t_{d y n, 0}$.

Fig. 10: Histograms of the cosine of the angle between $\mathbf{v}$ and $\mathbf{B}$. In experiment A1 (left panel) there is a partial alignment, while in experiment B1 (right panel) the two fields are mainly perpendicular to each other. 


\begin{tabular}{|c|c|c|c|c|c|c|c|c|}
\hline Model name & $\mathcal{M}_{A}$ & $b_{0}$ & $\beta$ & $\mathcal{M}$ & ambi & snapshots & Remarks & File name \\
\hline A1 & $\mathbf{1 0 . 6}$ & 0.5 & 5.33 & 5.3 & 0.0 & 31 & decaying & run_1 \\
Aa1 & $\mathbf{1 0 . 6}$ & 0.5 & 5.33 & 5.3 & 0.25 & 31 & decaying & run_11 \\
Aa2 & $\mathbf{1 0 . 6}$ & 0.5 & 5.33 & 5.3 & 0.50 & 30 & decaying & run_8 \\
B1 & $\mathbf{0 . 8}$ & 5.0 & 0.05 & 3.8 & 0.0 & 31 & decaying & run_3 \\
B2 & $\mathbf{1 . 1}$ & 5.0 & 0.05 & 5.4 & 0.0 & 11 & decaying & run_16 \\
B3 & $\mathbf{2 . 7}$ & 2.0 & 0.33 & 5.4 & 0.0 & 21 & decaying & run_17 \\
Ba1 & $\mathbf{0 . 8}$ & 5.0 & 0.05 & 3.8 & 0.50 & 14 & decaying & run_7 \\
Ba2 & $\mathbf{1 . 3}$ & 3.0 & 0.15 & 3.8 & 0.50 & 20 & decaying & run_12 \\
\hline Ad1 & $\mathbf{1 0 . 6}$ & 1.0 & 1.33 & 10.6 & 0.0 & 30 & driven & run_18 \\
Ad2 & $\mathbf{2 8 . 3}$ & 1.0 & 1.33 & 28.3 & 0.0 & 31 & driven & run_19 \\
Bd1 & $\mathbf{0 . 9}$ & 10.0 & 0.01 & 8.8 & 0.0 & 23 & driven & run_21 \\
Bd2 & $\mathbf{1 . 2}$ & 3.6 & 0.10 & 6.8 & 0.0 & 31 & driven & run_23 \\
Bd3 & $\mathbf{1 . 5}$ & 7.2 & 0.03 & 10.7 & 0.0 & 30 & driven & run_22 \\
Bd4 & $\mathbf{2 . 0}$ & 7.0 & 0.03 & 14.2 & 0.0 & 15 & driven & run_20 \\
\hline
\end{tabular}

Table 1: 


\begin{tabular}{|c|c|c|c|c|c|}
\hline Model name & $\sigma_{v} /(\mathrm{km} / \mathrm{s})$ & $l / \mathrm{pc}$ & $\tau_{\text {dyn }} /\left(10^{6} \mathrm{yr}\right)$ & $\langle n\rangle /\left(100 \mathrm{H}_{2} \mathrm{~cm}^{-3}\right)$ & $B_{0} / \mu \mathrm{G}$ \\
\hline $\mathrm{A} 1$ & 1.3 & 1.8 & 1.3 & 11 & 2.6 \\
$\mathrm{Aa} 1$ & 1.3 & 1.8 & 1.3 & 11 & 2.6 \\
$\mathrm{Aa} 2$ & 1.3 & 1.8 & 1.3 & 11 & 2.6 \\
$\mathrm{~B} 1$ & 0.9 & 0.9 & 0.9 & 22 & 36 \\
$\mathrm{~B} 2$ & 1.3 & 1.8 & 1.3 & 11 & 26 \\
$\mathrm{~B} 3$ & 1.3 & 1.8 & 1.3 & 11 & 10 \\
$\mathrm{Ba} 1$ & 0.9 & 0.9 & 0.9 & 22 & 36 \\
$\mathrm{Ba} 2$ & 0.9 & 0.9 & 0.9 & 22 & 22 \\
\hline $\mathrm{Ad} 1$ & 2.6 & 7.0 & 2.6 & 2.8 & 2.6 \\
$\mathrm{Ad} 2$ & 7.1 & 50 & 7.1 & 0.4 & 1.0 \\
$\mathrm{Bd} 1$ & 2.2 & 4.8 & 2.2 & 4.1 & 31 \\
$\mathrm{Bd} 2$ & 1.7 & 2.9 & 1.7 & 6.9 & 15 \\
$\mathrm{Bd} 3$ & 2.7 & 7.1 & 2.7 & 2.8 & 19 \\
$\mathrm{Bd} 4$ & 3.5 & 13 & 3.5 & 1.6 & 14 \\
\hline
\end{tabular}

Table 2: 

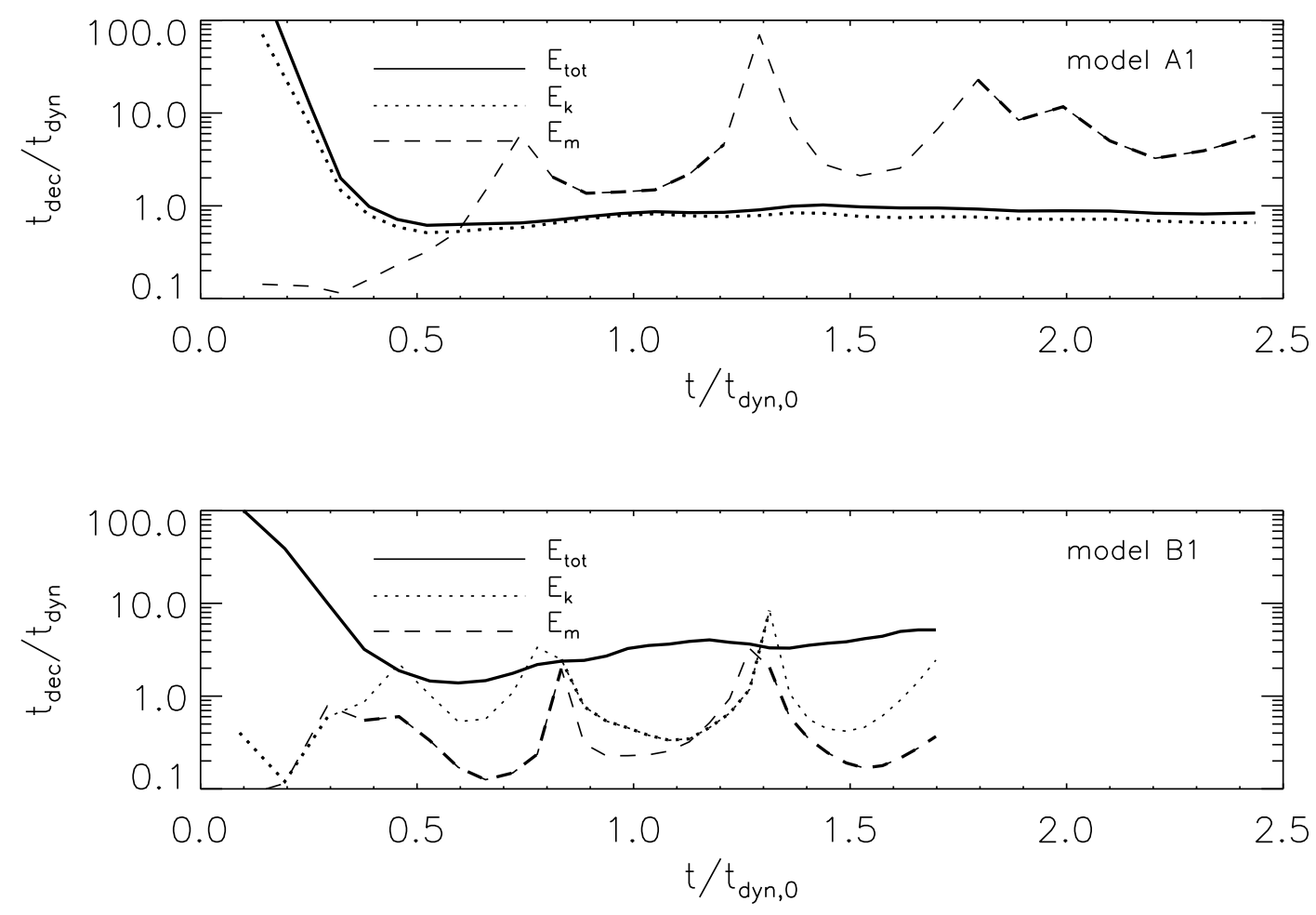

Fig. 1.- 

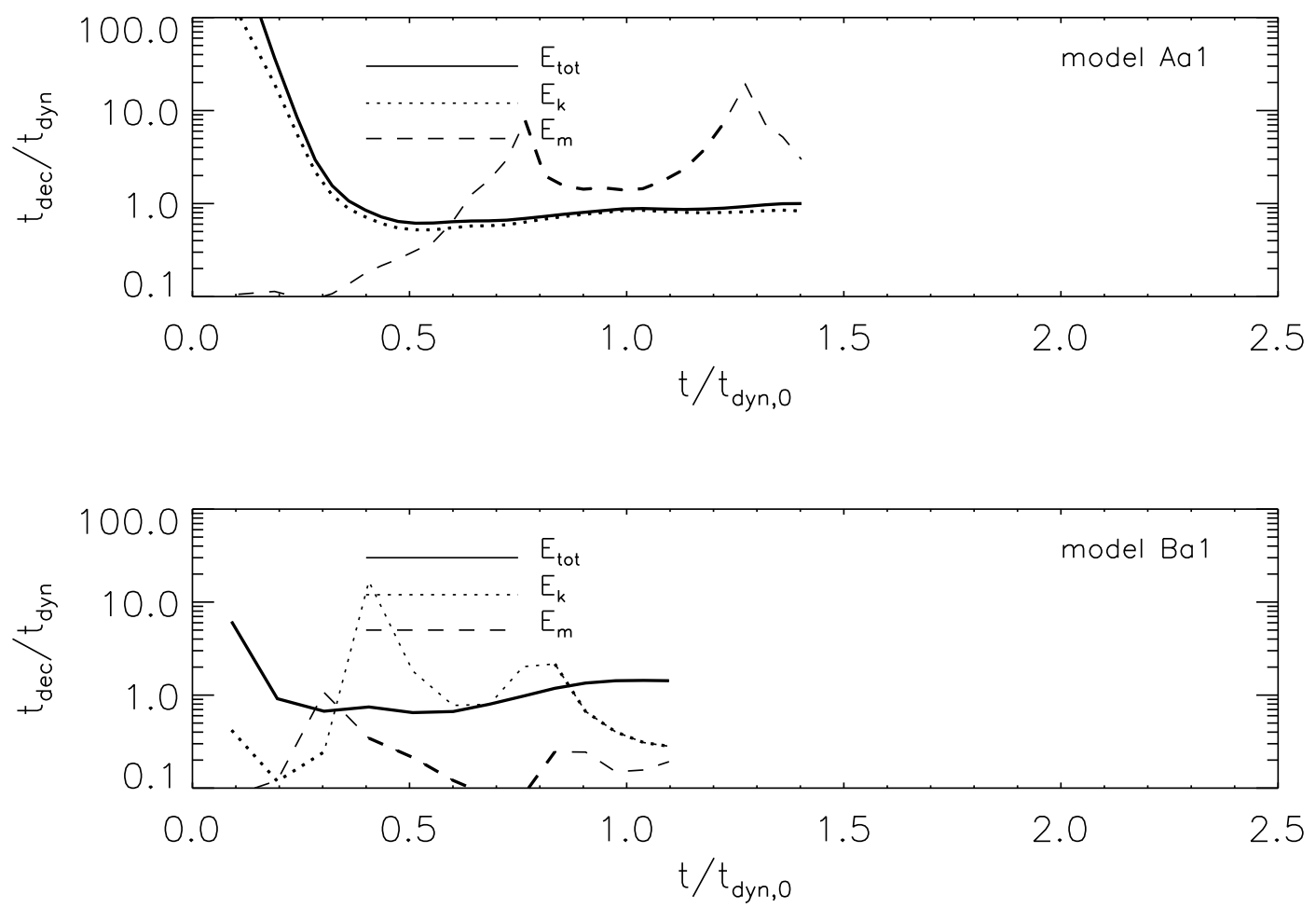

Fig. 2.- 


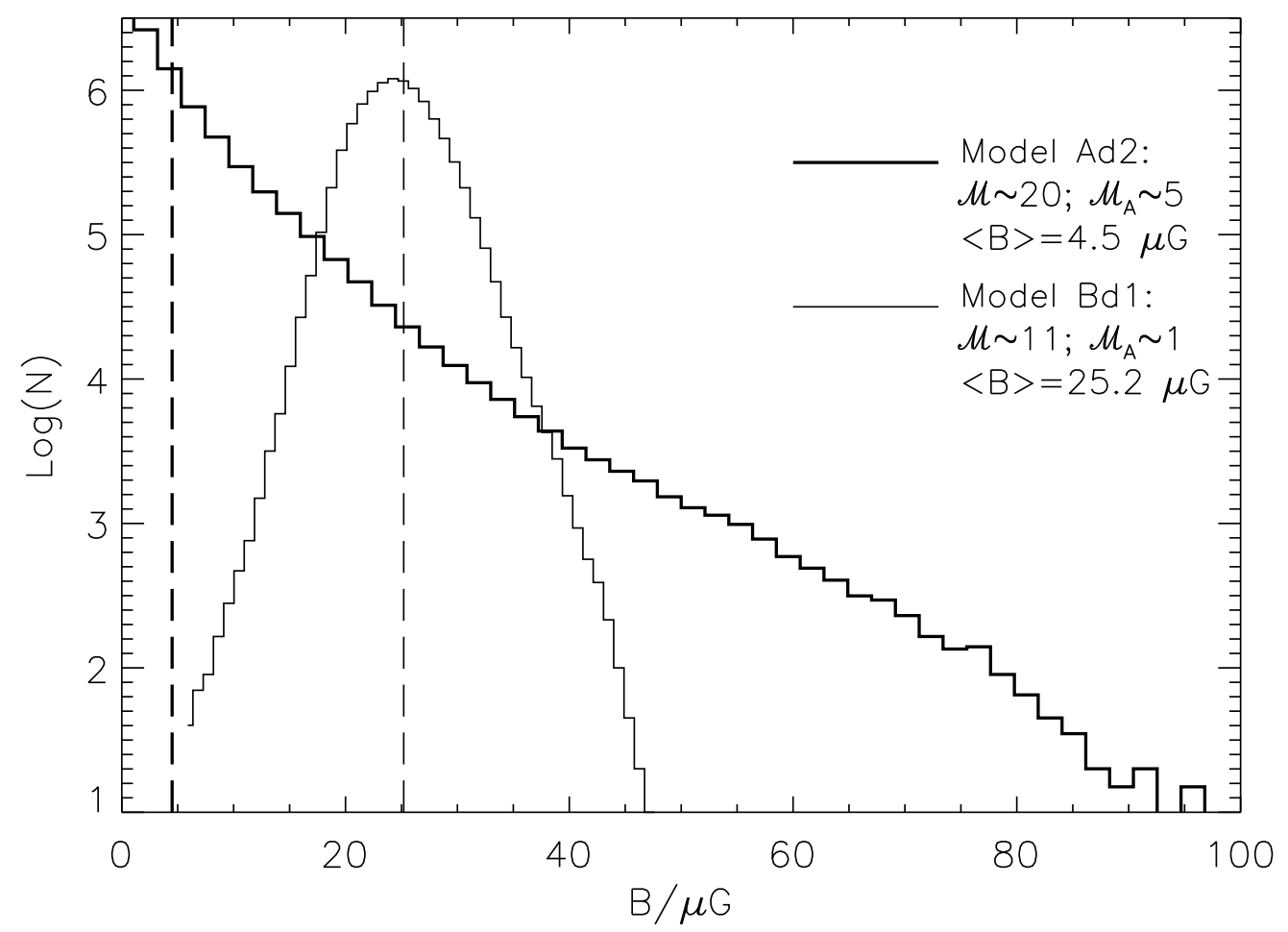

Fig. 3.- 

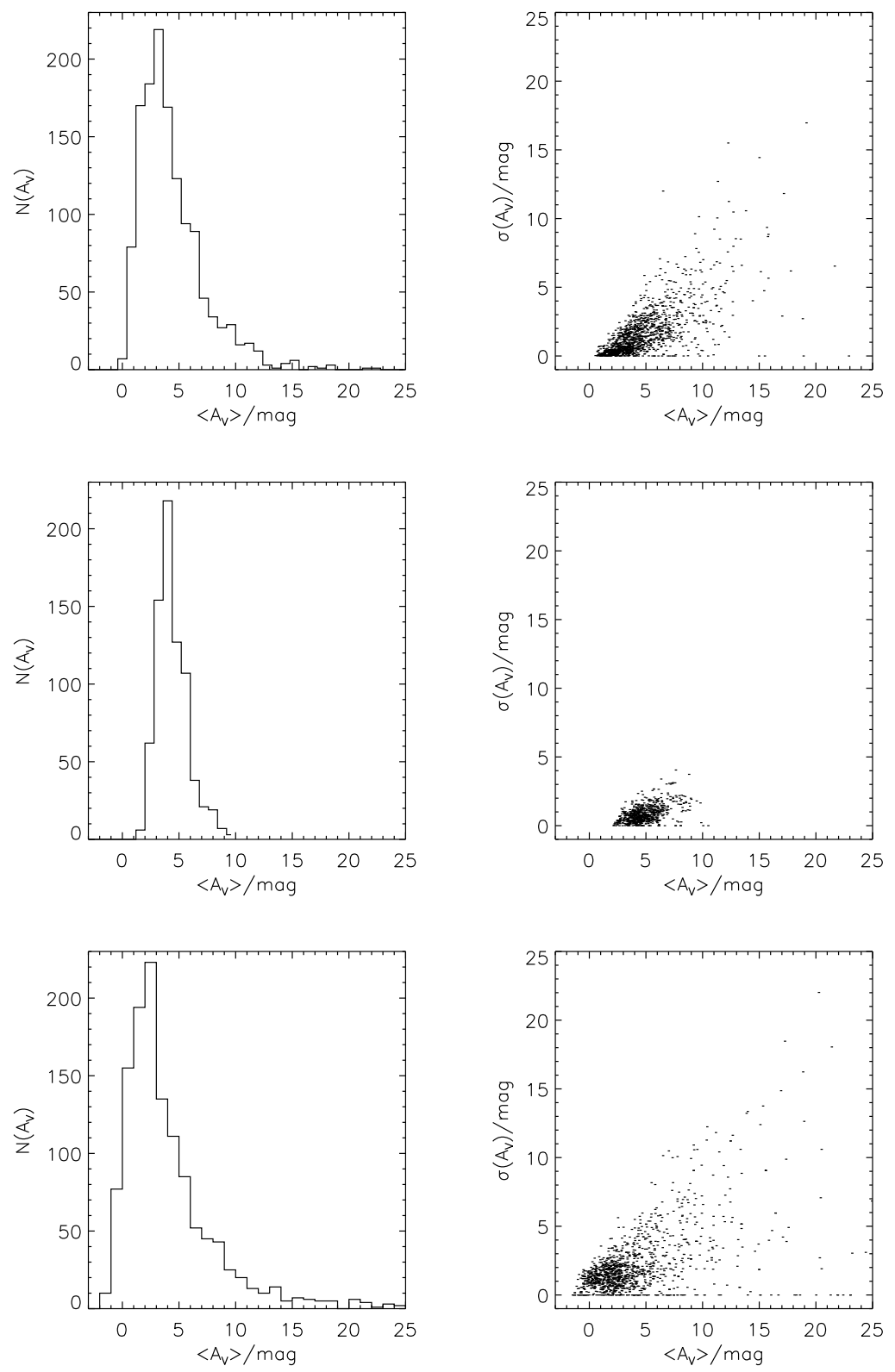

Fig. 4.- 


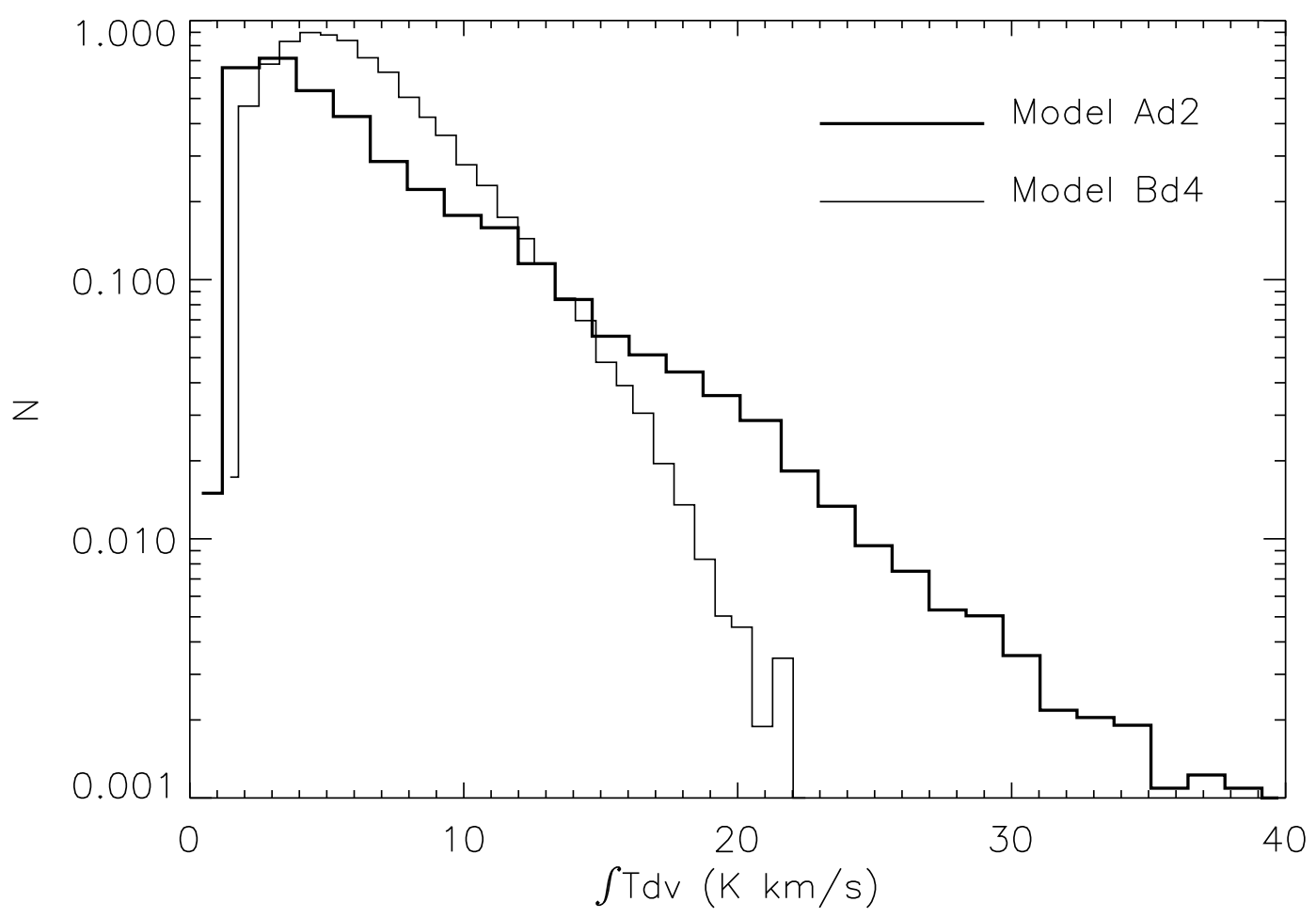

Fig. 5.- 

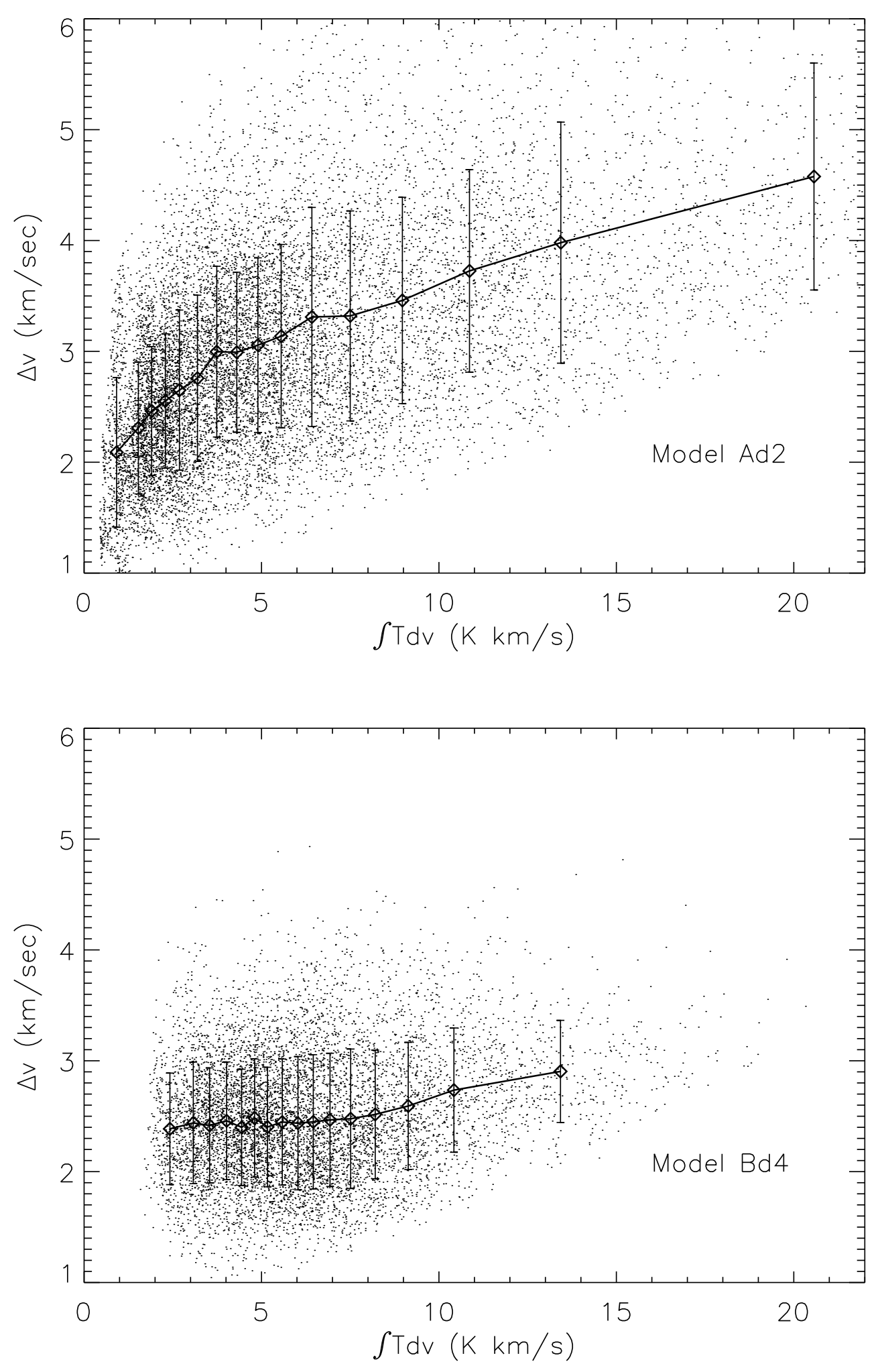

Fig. 6.- 


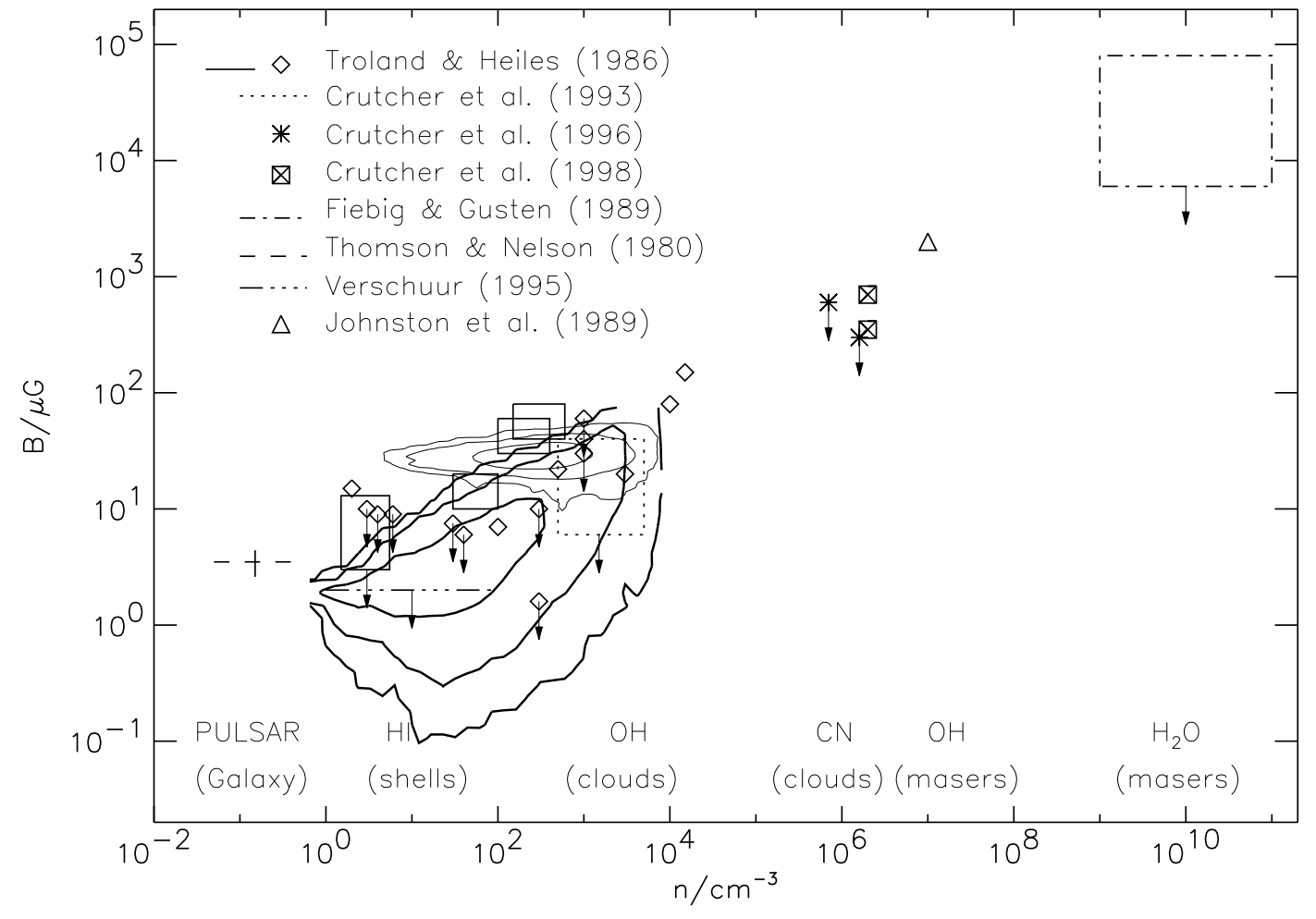

Fig. 7.- 

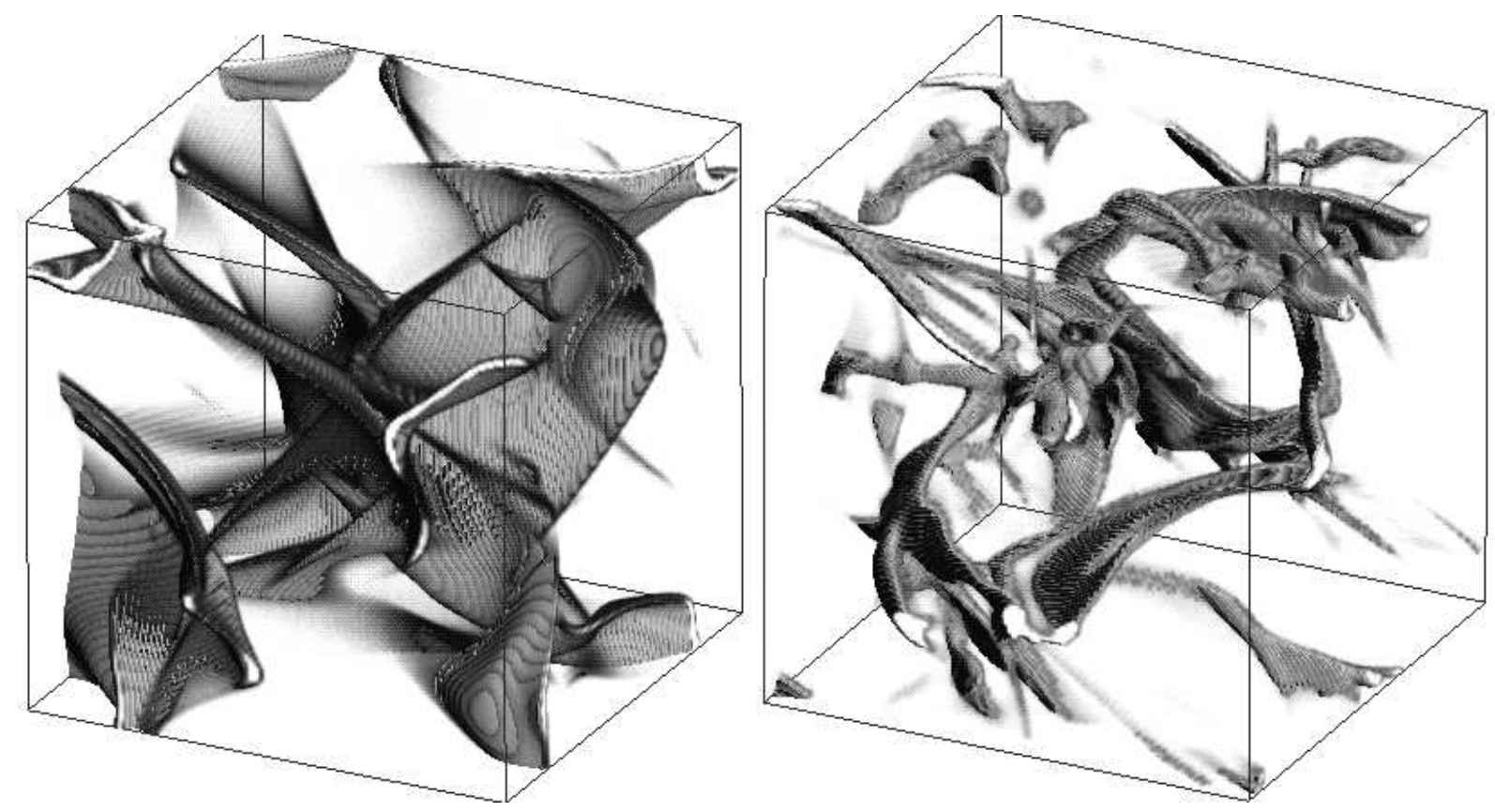

Fig. 8.- 


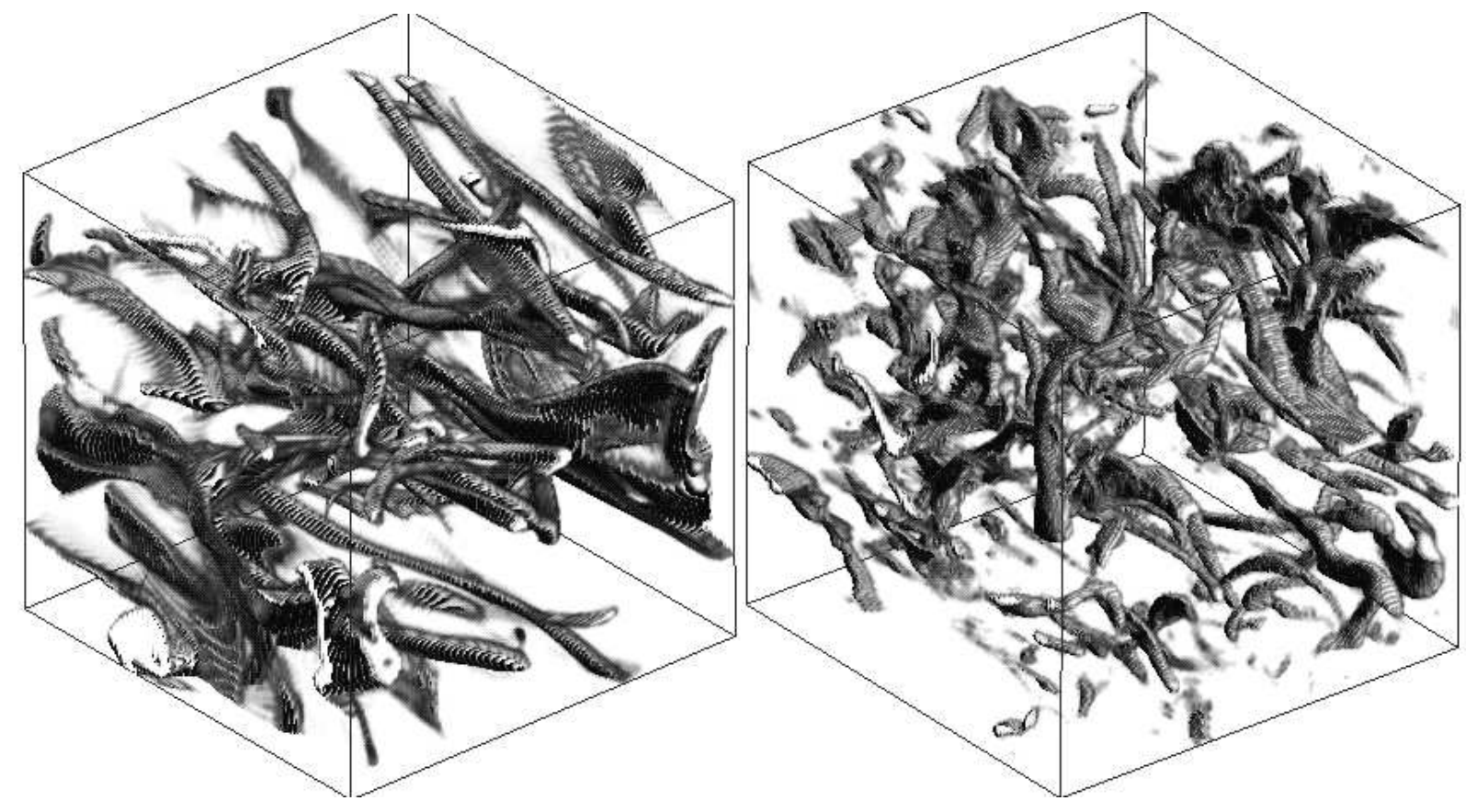

Fig. 9.- 

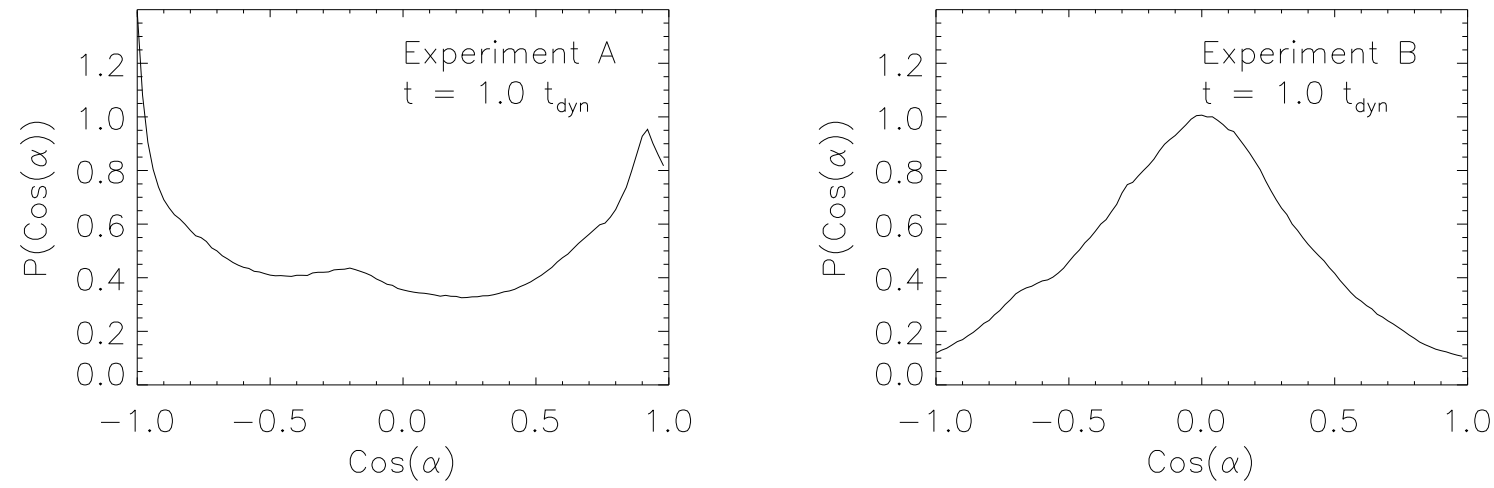

Fig. 10.- 